تأثير الكبريت والنتروجين وحامض الاسكوربيك في المحتوى المعدني لأشجار التفاح الفتية صنفي Anna

\title{
Vistabella $و$
}

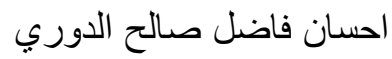 \\ قسم البستنة/كلية الزر اعة/ جامعة التكريت
}

\author{
جاسم محمد علوان الاعرجي \\ قسم البستنة و هندسة الحدائق/كلية الزر اعة واعة والغابات \\ جامعة الموصل
}

\section{الخلاصة}

اجريت تجربتين منفصلتين في حقول الفاكهة التابعه لكلية الزراعة والغابات/جامعة الموصل خلال

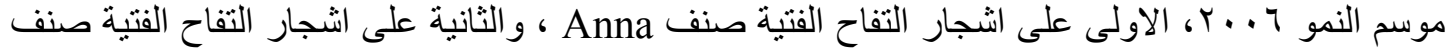

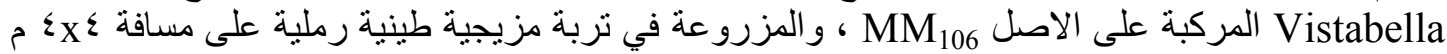

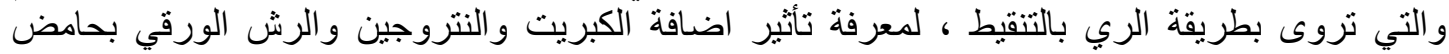

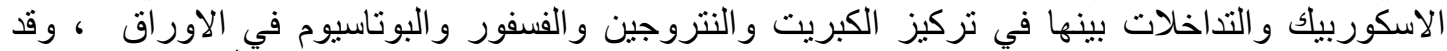

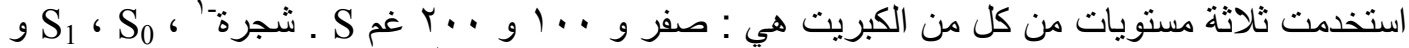

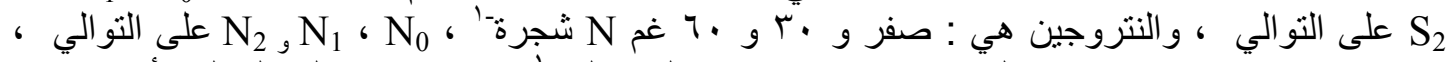

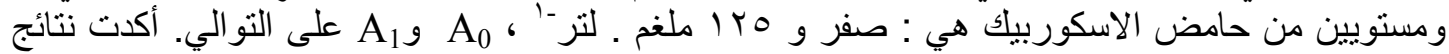

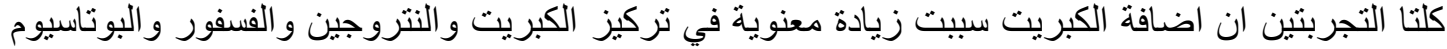

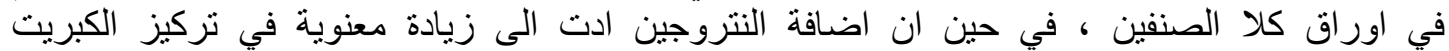

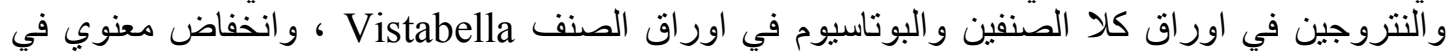

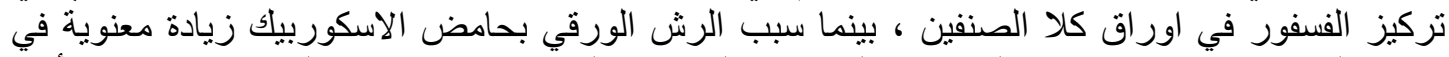

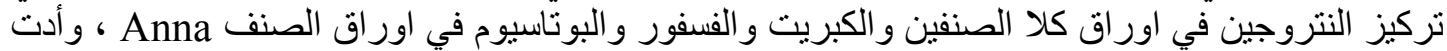

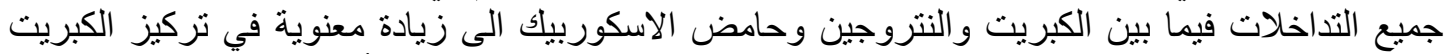

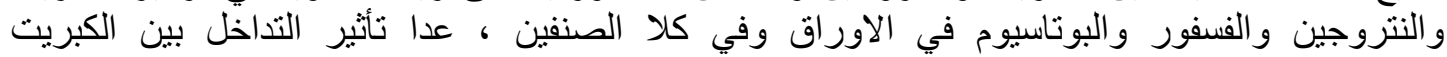

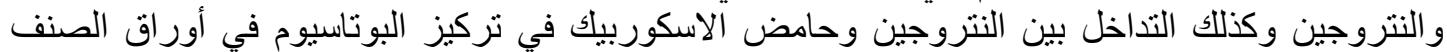

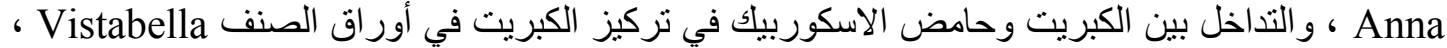

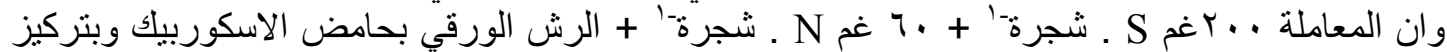

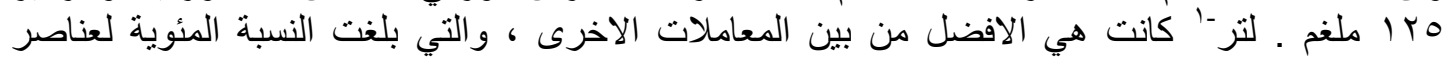

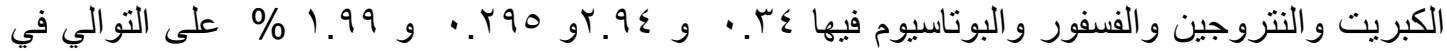

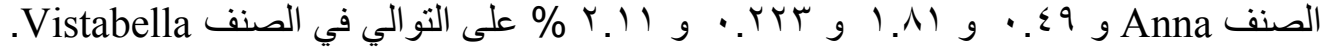

\section{المقدمة}

يحتل التفاح Malus domestica Borkh الذي يتبع العائلة الوردية Rosaceae مرنبة متُقدمة في

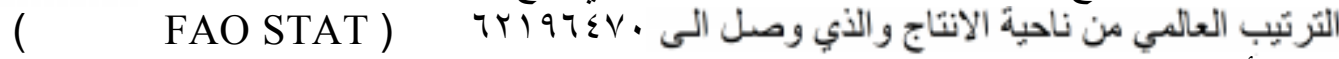

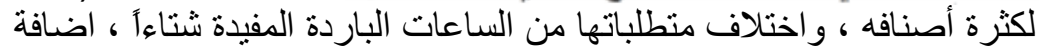

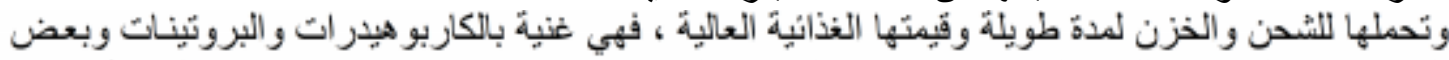

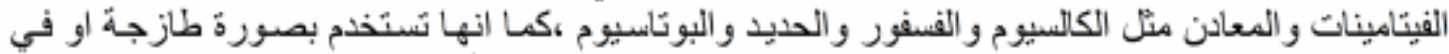

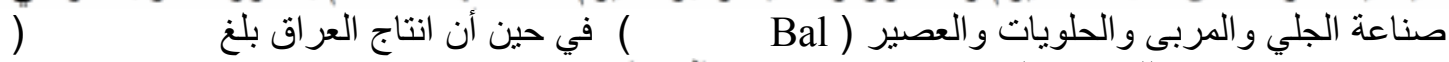

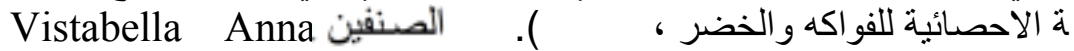

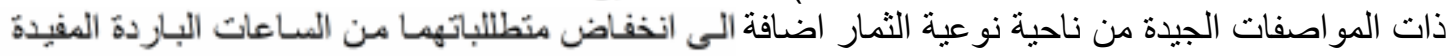

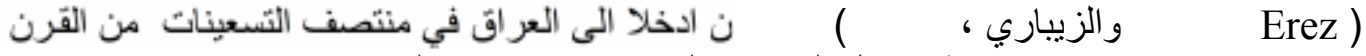

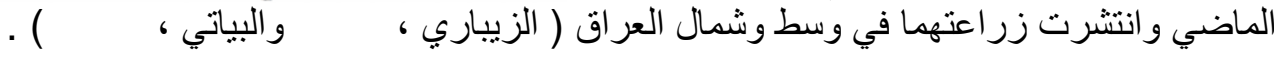

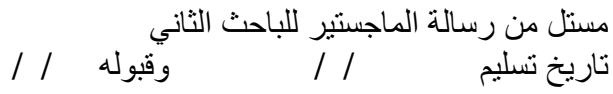




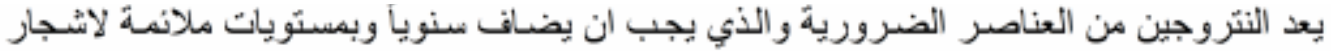

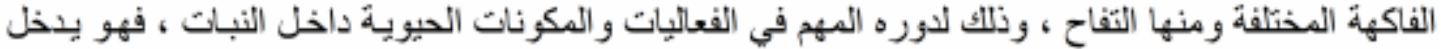

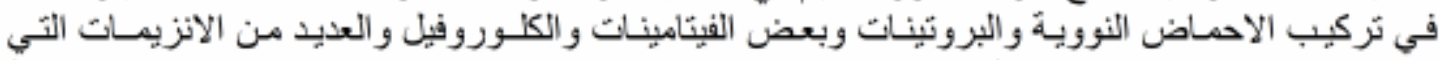

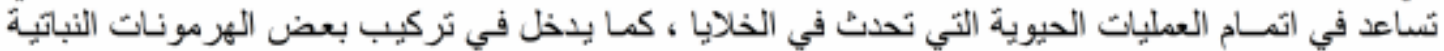

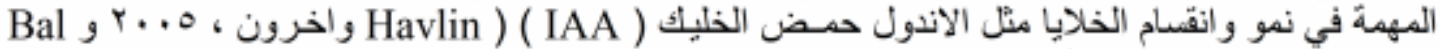

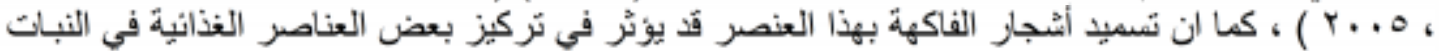

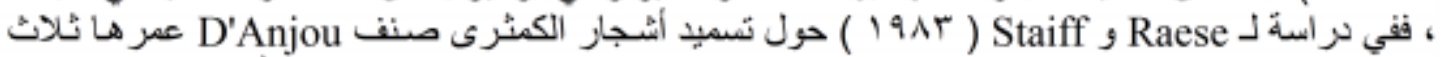

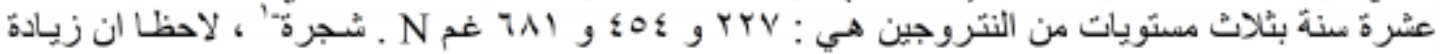

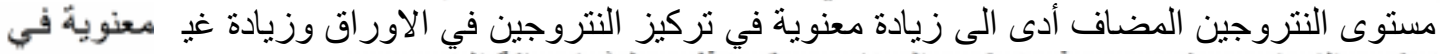

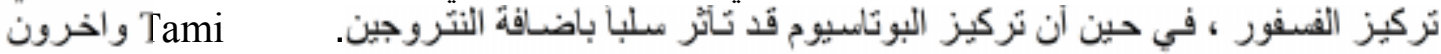

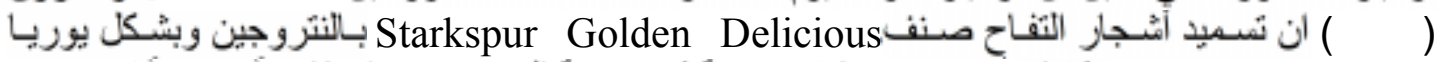

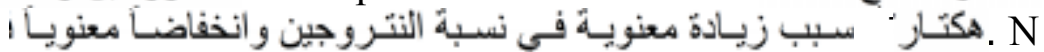

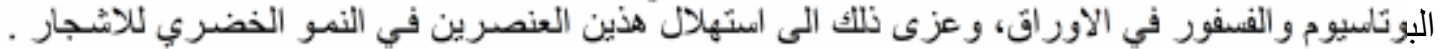

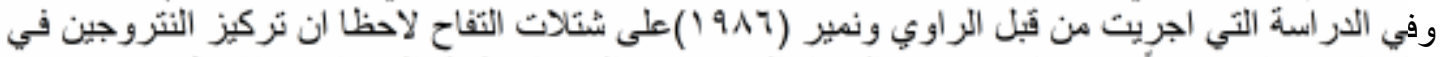

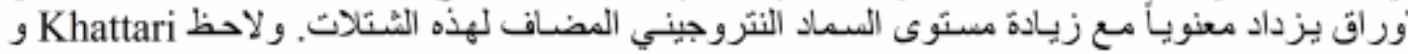
Shatat

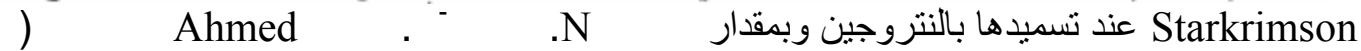

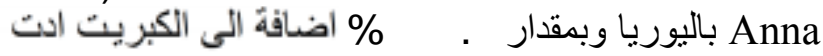

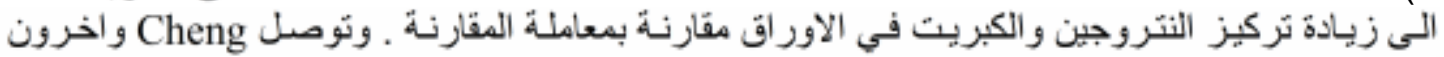

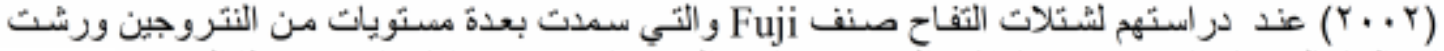

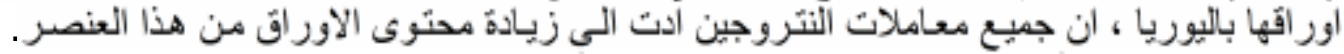

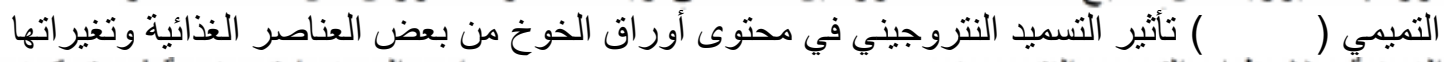

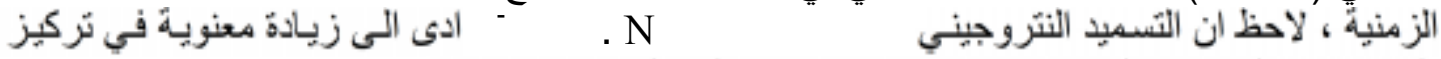

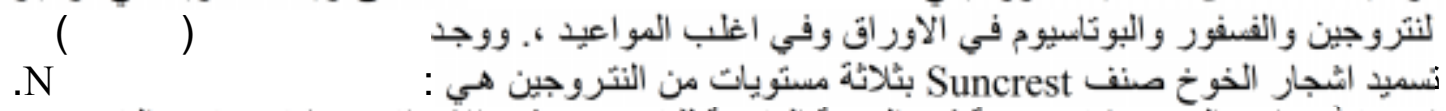

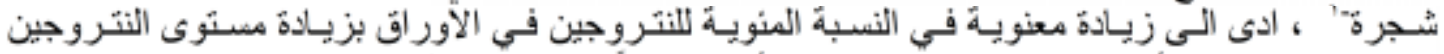

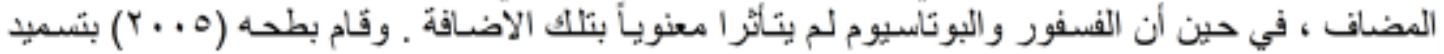

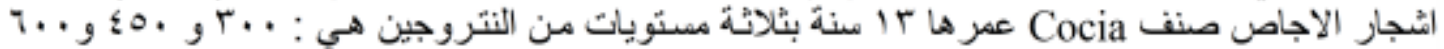

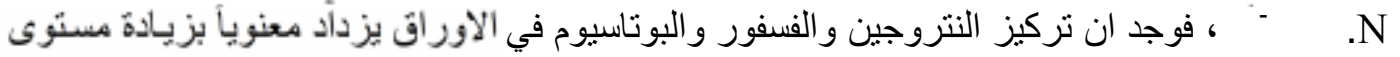

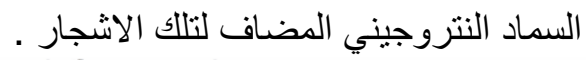

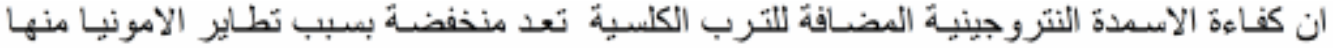

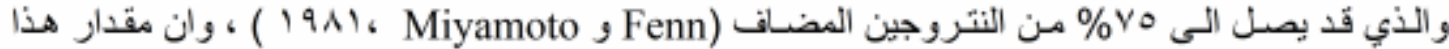

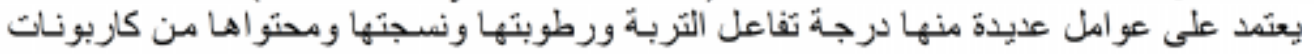

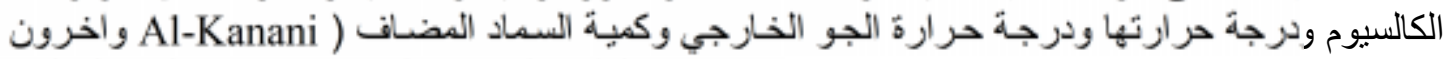

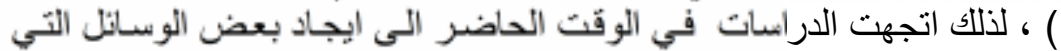

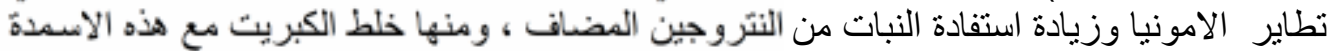

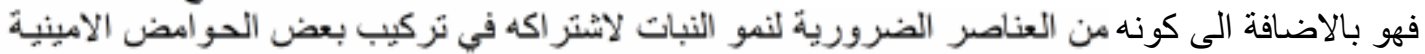
و والـ Cystine Cysteine

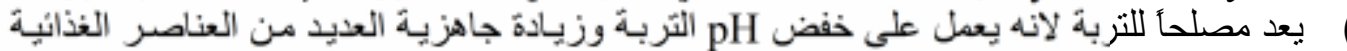

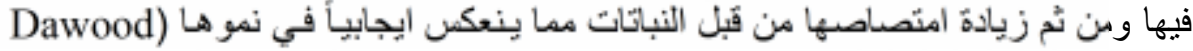

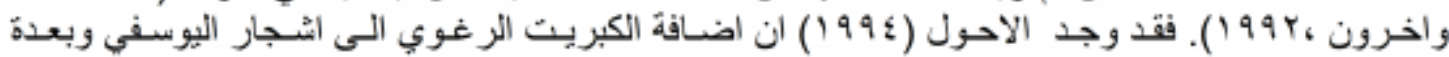

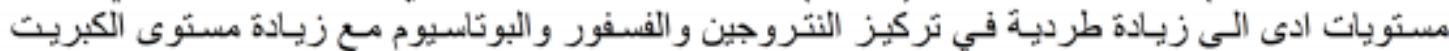

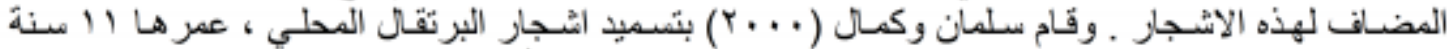

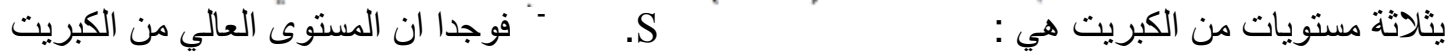

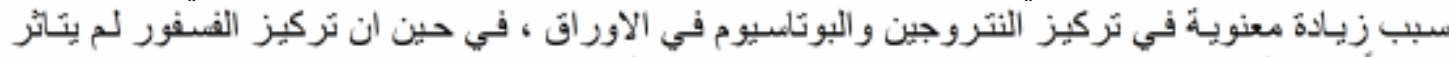

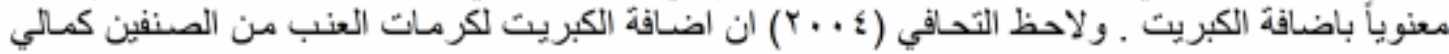




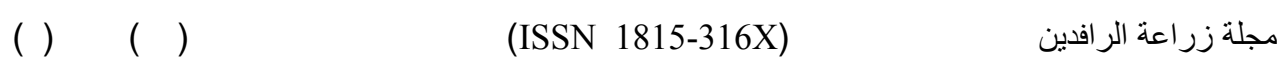

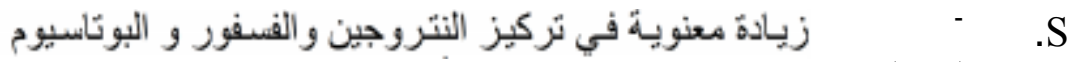

في اور اق كلا الصنفين مقارنة بمعاملتي المقارنة و

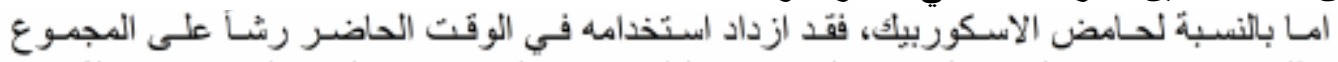

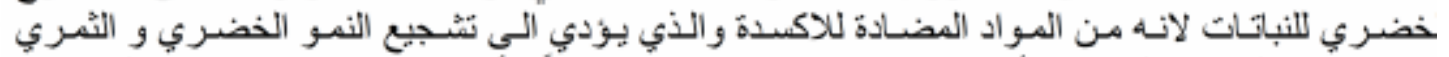

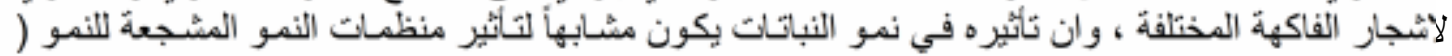
Ahmed

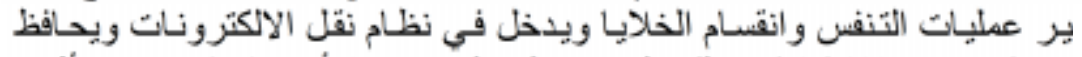

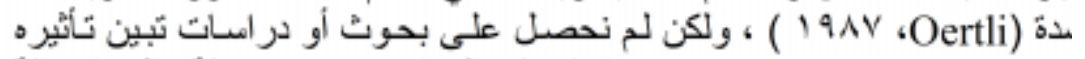

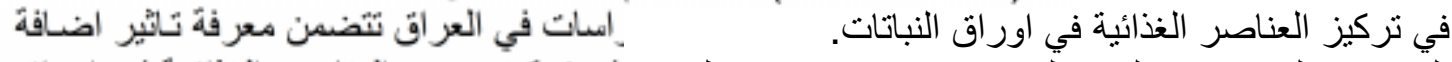

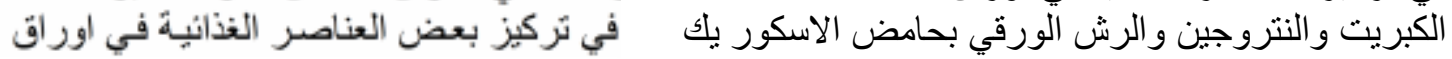

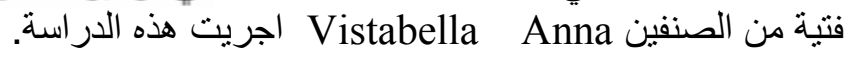

مواد البحث وطرائقه

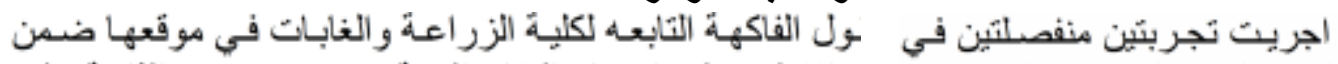

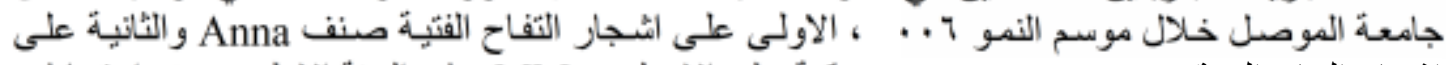

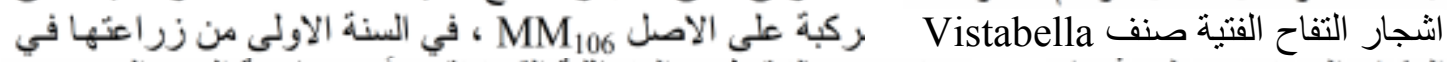

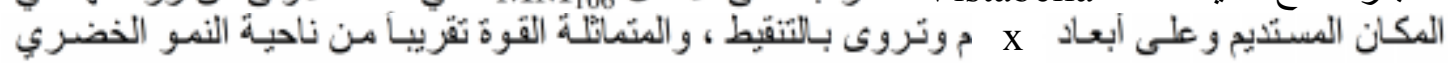

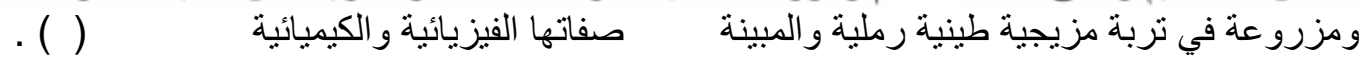

\begin{tabular}{|c|c|}
\hline \multicolumn{2}{|l|}{ القيمة } \\
\hline . & وصبل الكهربائي (ديسي سيمنز. - ) \\
\hline \multicolumn{2}{|r|}{ 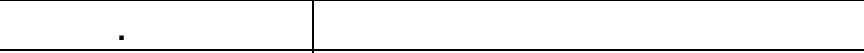 } \\
\hline . & المادة العضوية ) \\
\hline . & $. \quad)$ \\
\hline - & الطين \\
\hline . & الغرين \\
\hline \multicolumn{2}{|c|}{ مزيجية طينية رملية } \\
\hline & النتروجين الجاهز ( . ) \\
\hline & 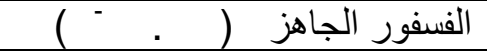 \\
\hline & البوتاسيوم الجاهز ( . - ـ ) \\
\hline . & البيكاربونات \\
\hline
\end{tabular}

* حللت التربة في مختبرات مديرية البحوث و الموارد المائية / نينوى

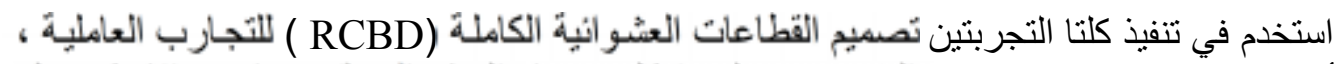

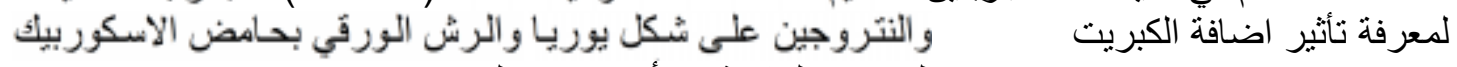

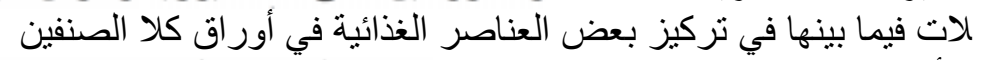

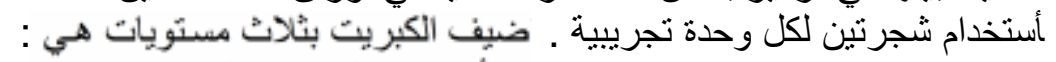

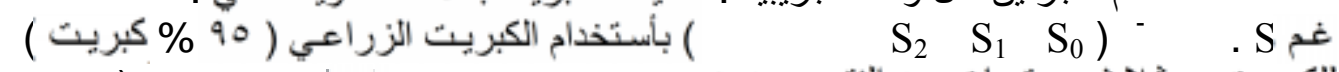
ل ل

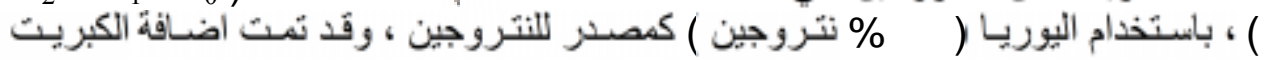
ونصف الكمية من النتروجين الى الوحدات التجريبية حسب المعاملات في الاسبوع الاول من نيسان من العام

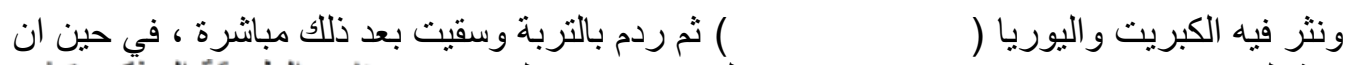

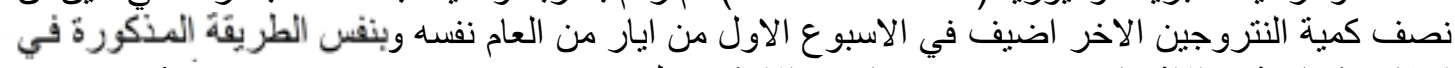
اعلاه ، كما رشت الاشجار بمستويين من حامض الاسكوربيك هي : 


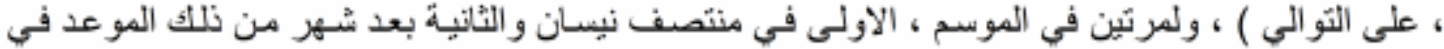

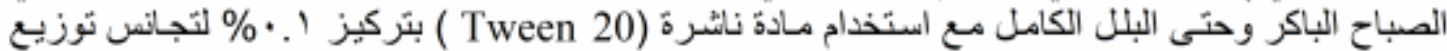

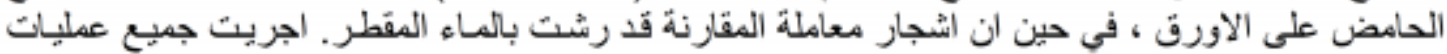

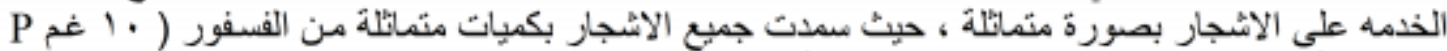

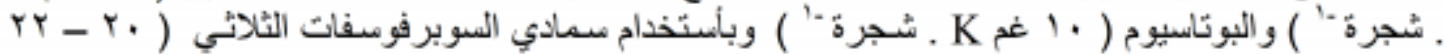

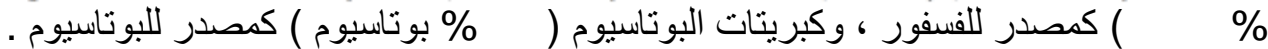

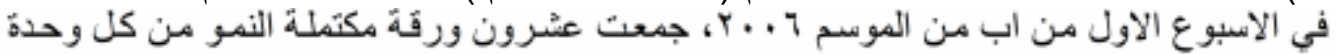

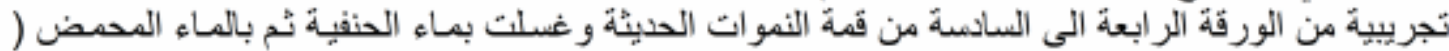

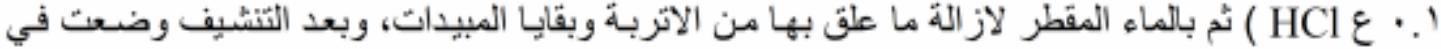

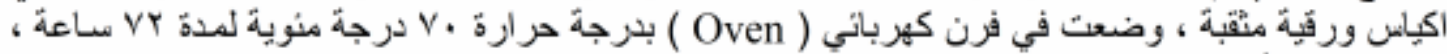

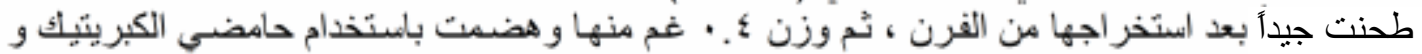

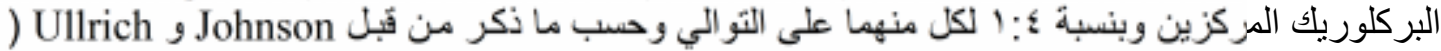

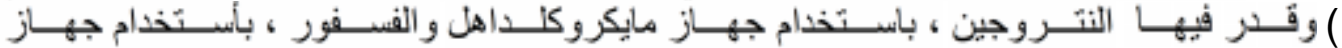
Spectrophotometer Raghupathi Bhargava بحامضي النتريك و البركلوريك وبنسبة : لكل منهما على الثو الي وقدر فيها الكبريت بالطريقة اللونية بعد

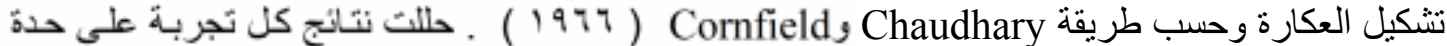

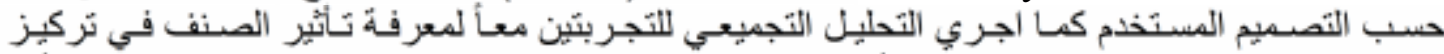

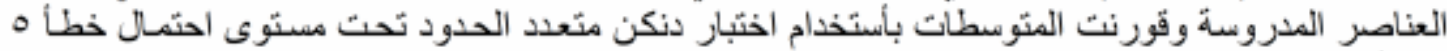
. ( SAS ) SAS

\section{النتائج والمناقشة}

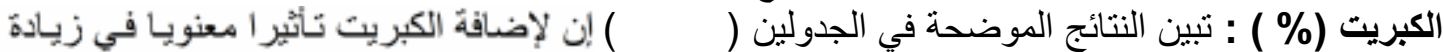

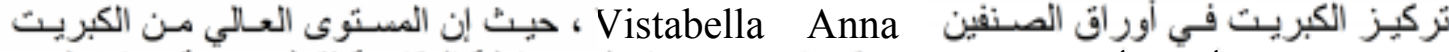

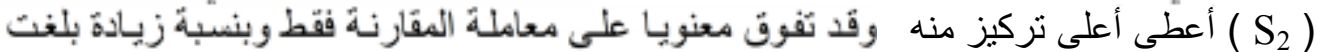

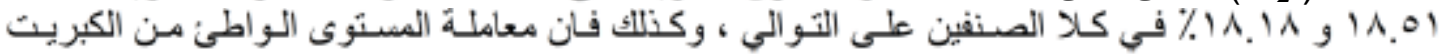

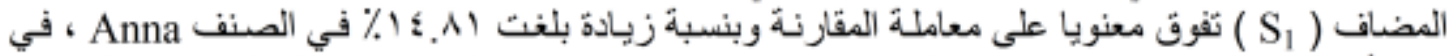

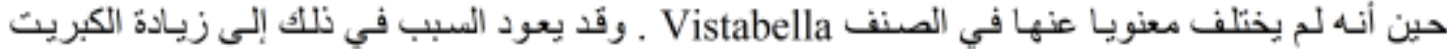

$$
\text { الجاهز في الثزبة نتيجة لإضافة الكبريث إلبها ( Sahu Intodia }
$$

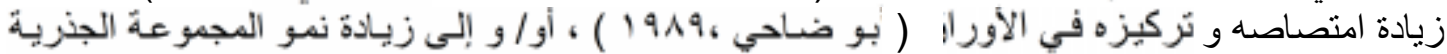

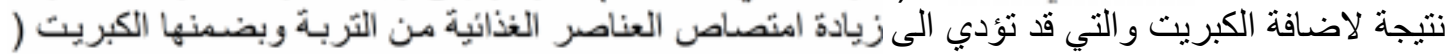

وكان للتسميد النتروجيني تأثبر معنوي في زيادة تركيز الكبريت في أوراق الصنفين ، وان المبن المستوى

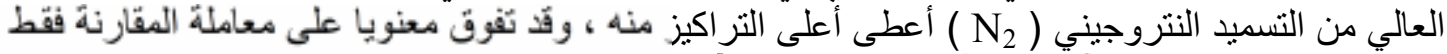

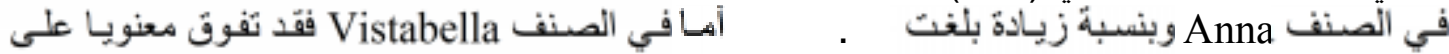

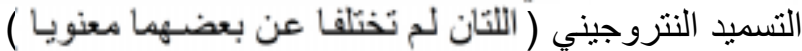

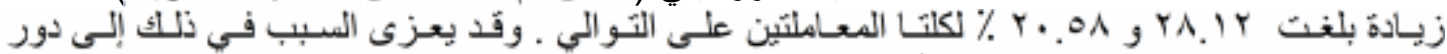

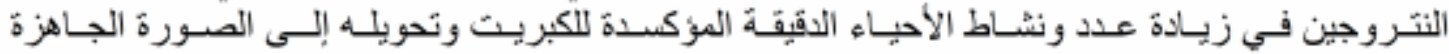

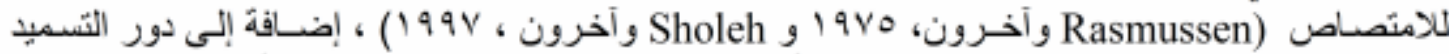

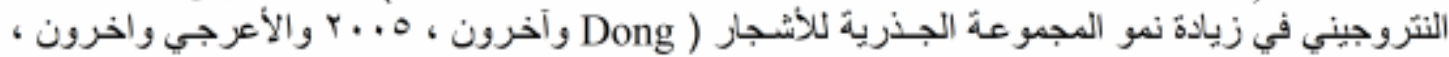

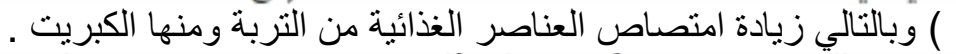

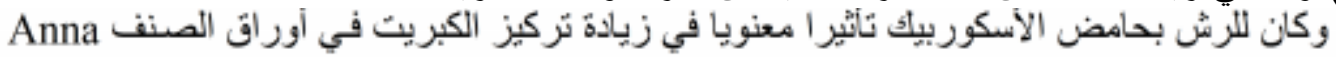

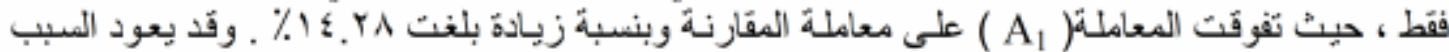

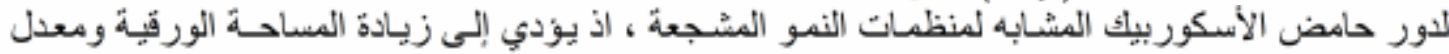

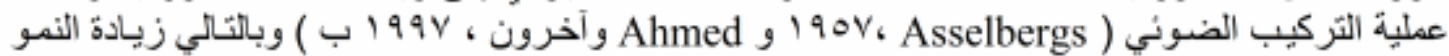

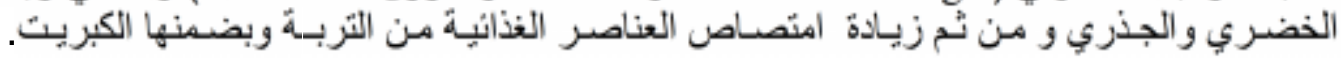



( ) ( )

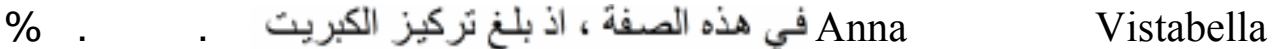

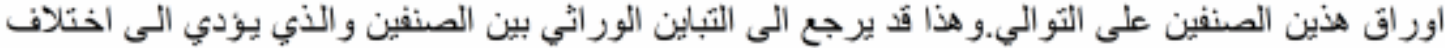

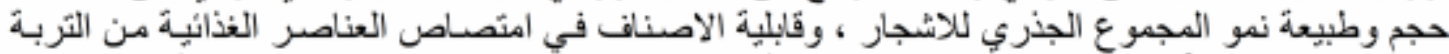

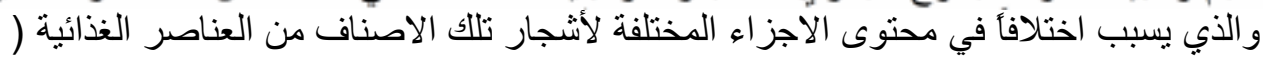
و ادت جميع التذاخلات بين الكبريت و النتروجين وحامض الاسكوربيك الى زيادة معنويـة في تركيز

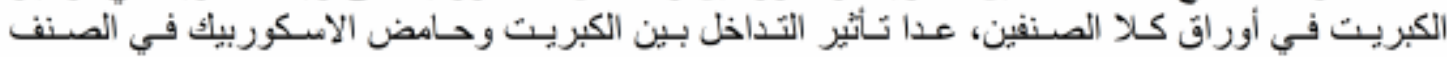

.Vistabella

( ): تأثثر الكبريت والنتروجين وحامض الأسكوربيك وتداخلاتهـا في تركيز الكبريت ( )

\begin{tabular}{|c|c|c|c|c|c|c|}
\hline \multirow[b]{3}{*}{ ين } & & & & nna & التفاح الفن & \\
\hline & \multirow{2}{*}{$\mathrm{N} \times \mathrm{A}$} & \multicolumn{3}{|c|}{ الكبريت ( S ) } & \multirow{2}{*}{ الأسكوربي (A) } & \multirow{2}{*}{$\begin{array}{c}\text { النتروجين } \\
\text { ( N ) }\end{array}$} \\
\hline & & $\mathrm{S}_{2}$ & $\mathrm{~S}_{1}$ & $\mathrm{~S}_{0}$ & & \\
\hline \multirow{2}{*}{-} & . & - & - & - & $\mathrm{A}_{0}$ & \multirow[t]{2}{*}{$\mathrm{N}_{0}$} \\
\hline & . & - & . & - & $\mathrm{A}_{1}$ & \\
\hline \multirow[t]{2}{*}{. } & . & - & - & - & $\mathrm{A}_{0}$ & \multirow[t]{2}{*}{$\mathrm{N}_{1}$} \\
\hline & . & . & - & - & $\mathrm{A}_{1}$ & \\
\hline \multirow[t]{2}{*}{. } & . & ه- & $ه-$ & هـ و & $\mathrm{A}_{0}$ & \multirow[t]{2}{*}{$\mathrm{N}_{2}$} \\
\hline & . & - & . & & $\mathrm{A}_{1}$ & \\
\hline & \multirow[b]{3}{*}{ الأسكوربيك } & - & . & & $\mathrm{N}_{0}$ & \multirow{3}{*}{$\mathrm{S} \times \mathrm{N}$} \\
\hline & & . & . & - & $\mathrm{N}_{1}$ & \\
\hline & & . & . & . & $\mathrm{N}_{2}$ & \\
\hline & 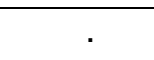 & . & . & . & $\mathrm{A}_{0}$ & \multirow{2}{*}{$\mathrm{S} \times \mathrm{A}$} \\
\hline & . & . & . & & $\mathrm{A}_{1}$ & \\
\hline & & & & & كبريت & متوسطا \\
\hline
\end{tabular}

* متوسطات كل مجمو عة المتبو عة بحروف مختلفة تدل على وجود فروقات معنوية بينها عند مستوى احتمال

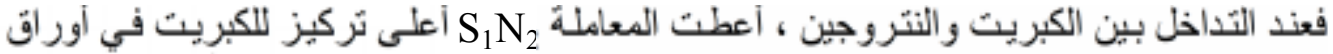
Anna

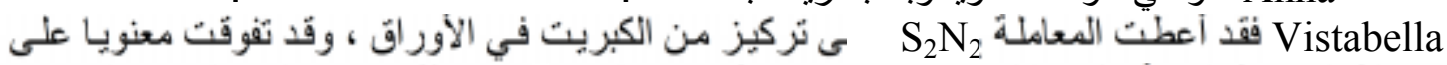

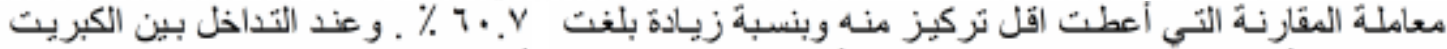

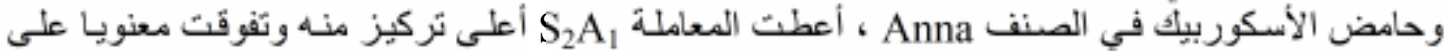

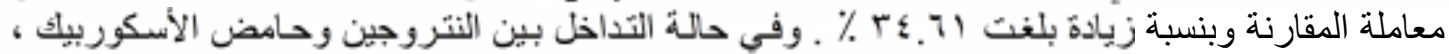

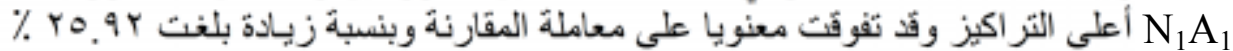

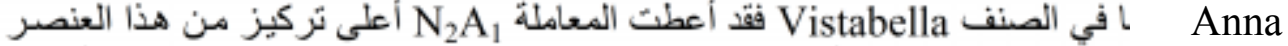

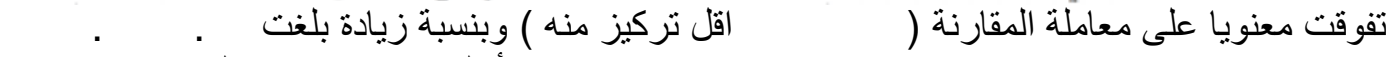

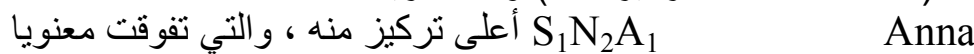

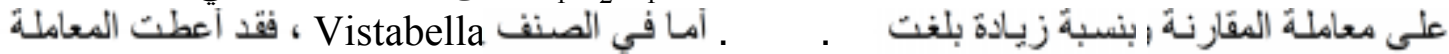

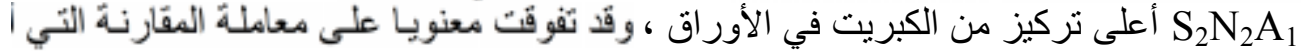

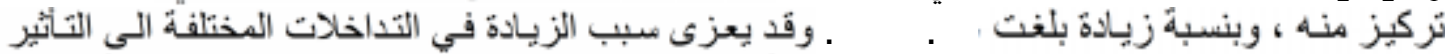

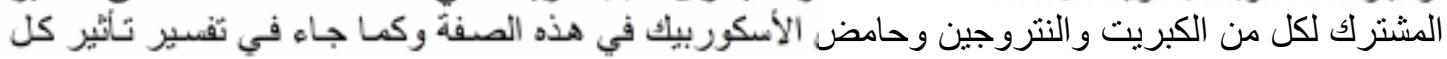

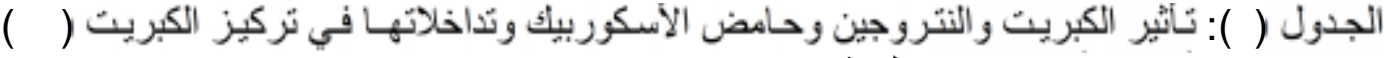

$$
\begin{aligned}
& \text { الفتية }
\end{aligned}
$$




\begin{tabular}{|c|c|c|c|c|c|c|}
\hline \multirow[b]{2}{*}{ ين } & \multirow{2}{*}{$\mathrm{N} \times \mathrm{A}$} & \multicolumn{3}{|c|}{ الكبريت ( S ) } & \multirow{2}{*}{ الأسكوربي } & \multirow{2}{*}{$\begin{array}{c}\text { النتروجين } \\
\text { ( N ) }\end{array}$} \\
\hline & & $\mathrm{S}_{2}$ & $\mathrm{~S}_{1}$ & $\mathrm{~S}_{0}$ & & \\
\hline \multirow[t]{2}{*}{. } & . &.- & . & . & $\mathrm{A}_{0}$ & \multirow[t]{2}{*}{$\mathrm{N}_{0}$} \\
\hline & . & - & - & . & $\mathrm{A}_{1}$ & \\
\hline \multirow[t]{2}{*}{. } & . & - & - & - & $\mathrm{A}_{0}$ & \multirow[t]{2}{*}{$\mathrm{N}_{1}$} \\
\hline & . & - &. & & $\mathrm{A}_{1}$ & \\
\hline \multirow[t]{2}{*}{. } & . & - & . & - & $\mathrm{A}_{0}$ & \multirow[t]{2}{*}{$\mathrm{N}_{2}$} \\
\hline & . & . & - & - & $\mathrm{A}_{1}$ & \\
\hline & \multirow[b]{3}{*}{ الأسكوربيك } & & . &. & $\mathrm{N}_{0}$ & \multirow{3}{*}{$\mathrm{S} \times \mathrm{N}$} \\
\hline & & - & . & . & $\mathrm{N}_{1}$ & \\
\hline & & . & . & . & $\mathrm{N}_{2}$ & \\
\hline & . & . & & . & $\mathrm{A}_{0}$ & \multirow[b]{2}{*}{$\mathrm{S} \times \mathrm{A}$} \\
\hline & . & . & & . & $\mathrm{A}_{1}$ & \\
\hline & & . & . & . & \multicolumn{2}{|c|}{ متو سطات الكبربت } \\
\hline
\end{tabular}

النتروجين ( \% ) : يتضح من النتائج في الجدولين ( 0 و 7 ) ) إن إضافة الكبريث أدت إلى زيادة معنوية في

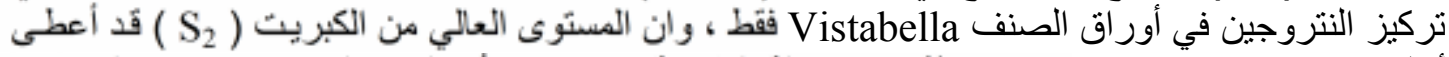

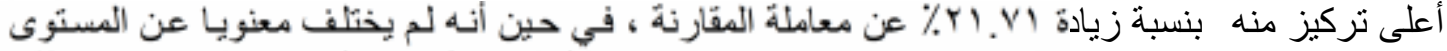

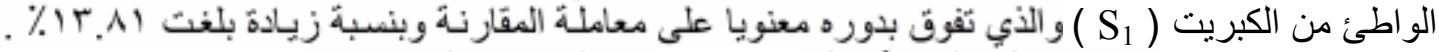

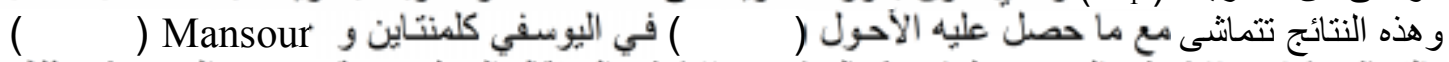

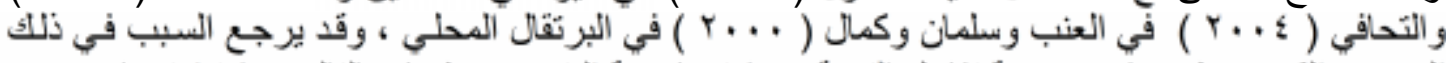

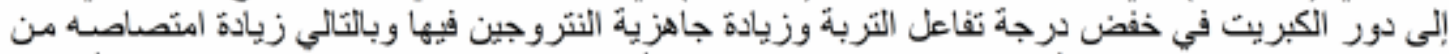

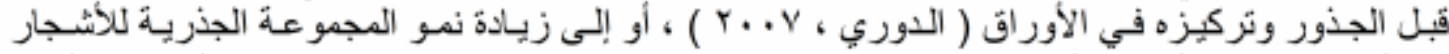

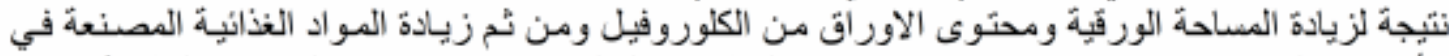

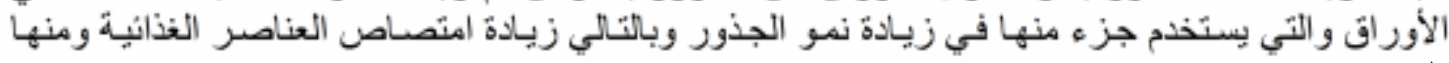

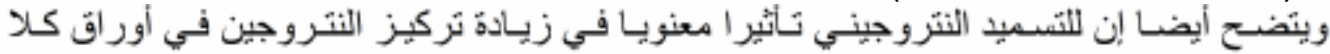

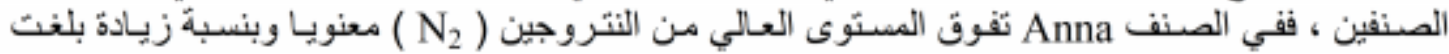

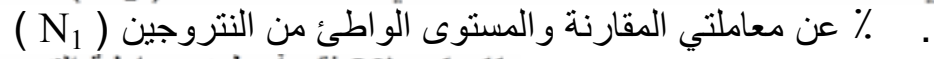

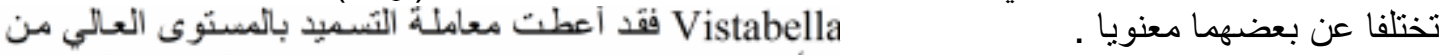

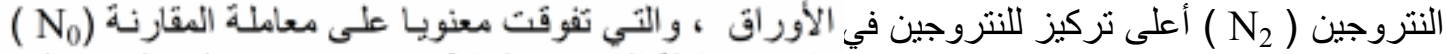

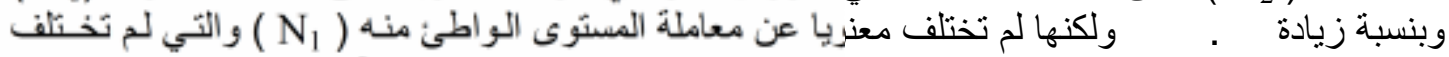

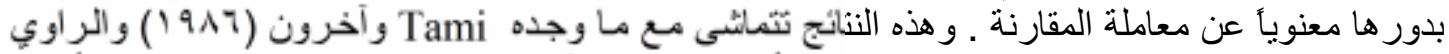

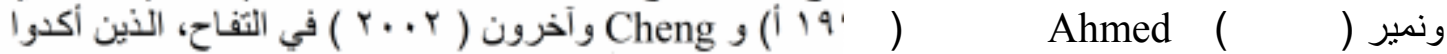

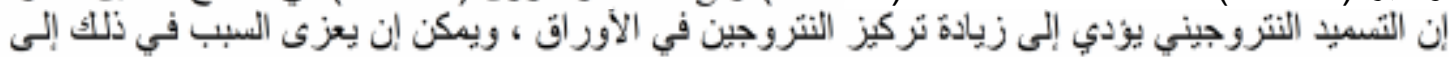

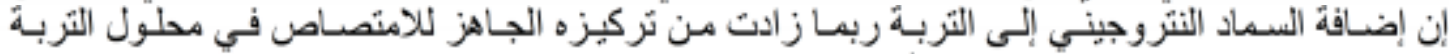

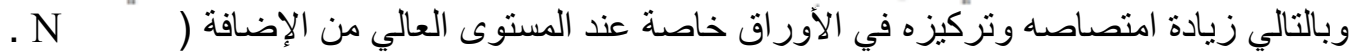

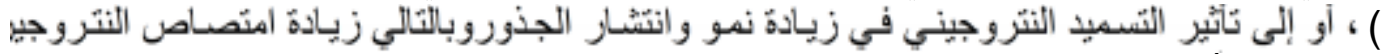

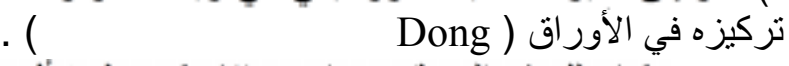

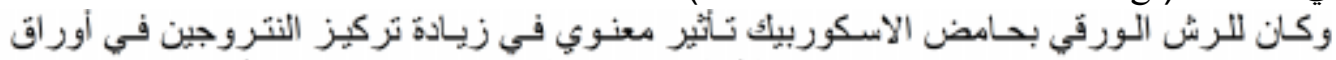

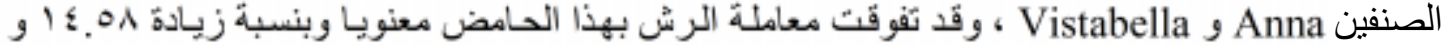

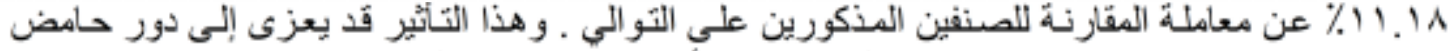

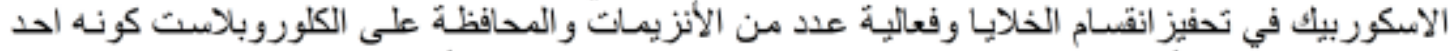

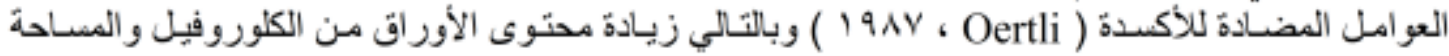

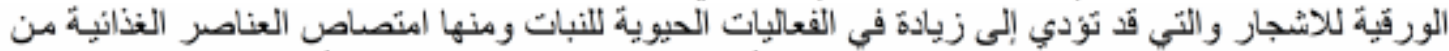

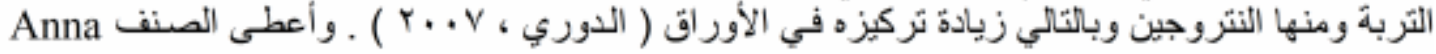



$($ )
(ISSN 1815-316X)
مجلة زر اعة الر افدين

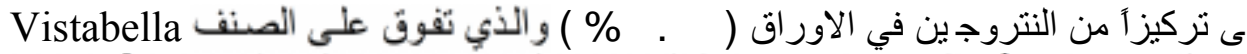

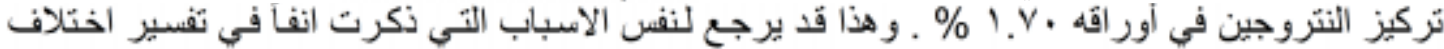

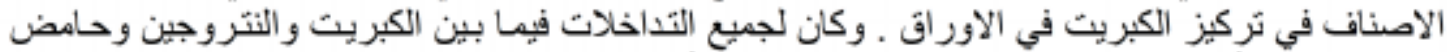

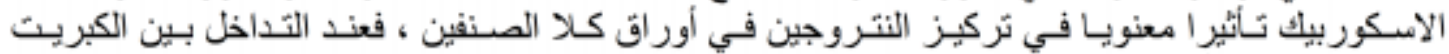

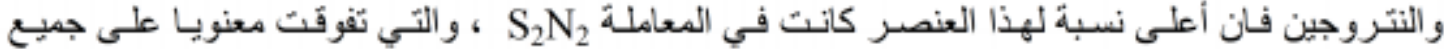

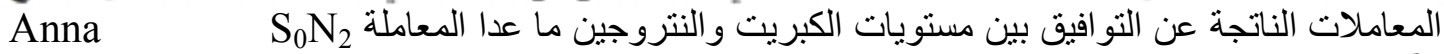

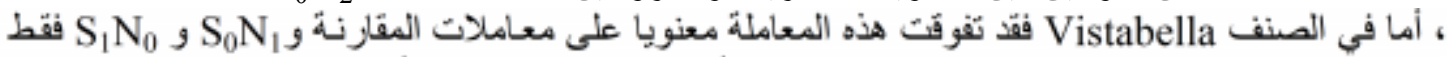

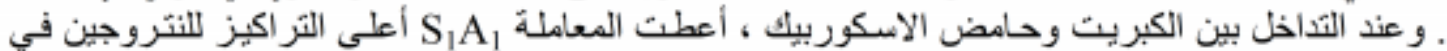

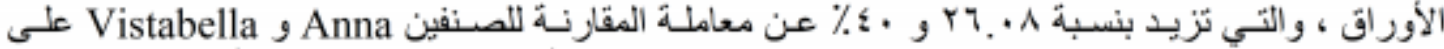

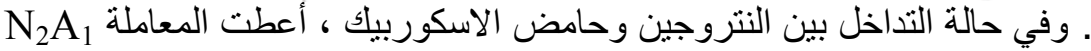

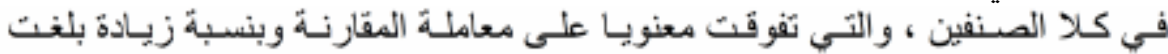

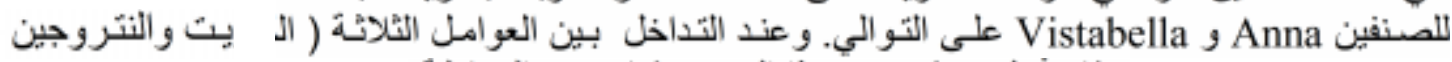

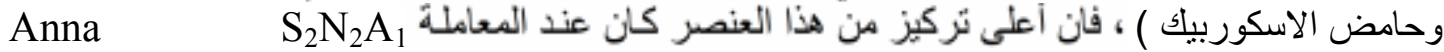

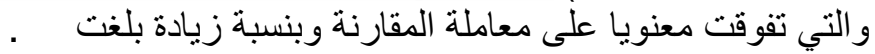

( ) أثثير الكبريت والنتروجين وحامض الأسكوربيك وتداخلاتها في نركيز النتروجين ( ) Anna A الفتية

\begin{tabular}{|c|c|c|c|c|c|c|}
\hline \multirow{2}{*}{ النتروجين } & \multirow{2}{*}{$\mathrm{N} \times \mathrm{A}$} & \multicolumn{3}{|c|}{ الكبريت ( S ) } & \multirow[b]{2}{*}{$\begin{array}{r}\text { الأسكوربي } \\
\text { (A) }\end{array}$} & \multirow{2}{*}{ النتروجين } \\
\hline & & $\mathrm{S}_{2}$ & $\mathrm{~S}$ & $\mathrm{~S}_{0}$ & & \\
\hline \multirow[t]{2}{*}{. } & . & - & - & . & $\mathrm{A}_{0}$ & \multirow[t]{2}{*}{$\mathrm{N}_{0}$} \\
\hline & . & - & - & ه- & $\mathrm{A}_{1}$ & \\
\hline \multirow[t]{2}{*}{. } & . & - & - & هـ & $\mathrm{A}_{0}$ & \multirow[t]{2}{*}{$\mathrm{N}_{1}$} \\
\hline & . & - & - & - & $\mathrm{A}_{1}$ & \\
\hline \multirow[t]{5}{*}{ - } & . & . & - & . & $\mathrm{A}_{0}$ & \multirow[t]{2}{*}{$\mathrm{N}_{2}$} \\
\hline & & & - & - & $\mathrm{A}_{1}$ & \\
\hline & \multirow[b]{3}{*}{ الأسكوربيك } & . & & . & $\mathrm{N}_{0}$ & \multirow{3}{*}{$\mathrm{S} \times \mathrm{N}$} \\
\hline & & . & & . & $\mathrm{N}_{1}$ & \\
\hline & & & & . & $\mathrm{N}_{2}$ & \\
\hline &. & . & & . & $\mathrm{A}_{0}$ & \multirow{2}{*}{$\mathrm{S} \times \mathrm{A}$} \\
\hline &. & . & . & . & $\mathrm{A}_{1}$ & \\
\hline & & 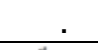 & & & كبريت & متوسطا. \\
\hline
\end{tabular}

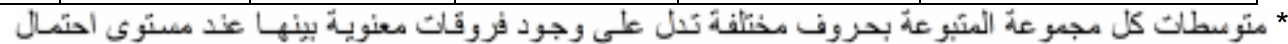

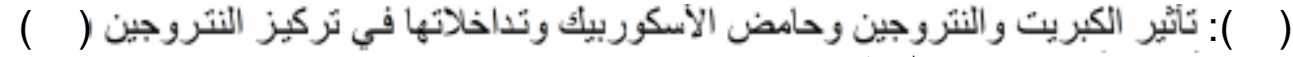

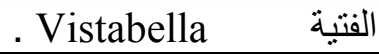

\begin{tabular}{|c|c|c|c|c|c|c|}
\hline \multirow[b]{2}{*}{ النتروجين } & \multirow[b]{2}{*}{$\mathrm{N} \times \mathrm{A}$} & \multicolumn{3}{|c|}{ الكبريت ( S ) } & \multirow{2}{*}{ الأسكوربي } & \multirow{2}{*}{ 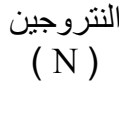 } \\
\hline & & $\mathrm{S}_{2}$ & $\mathrm{~S}_{1}$ & $\mathrm{~S}_{0}$ & & \\
\hline \multirow[t]{2}{*}{. } & . & . & . & 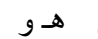 & $\mathrm{A}_{0}$ & \multirow[t]{2}{*}{$\mathrm{N}_{0}$} \\
\hline & . & هـ - & - & - & $A_{1}$ & \\
\hline \multirow[t]{2}{*}{. } & . & $ه-$ & - & - & $\mathrm{A}_{0}$ & \multirow[t]{2}{*}{$\mathrm{N}_{1}$} \\
\hline & . & هـ & . & هـ & $\mathrm{A}_{1}$ & \\
\hline \multirow[t]{2}{*}{. } & . & . & $\rightarrow-$ & - & $\mathrm{A}_{0}$ & \multirow[t]{2}{*}{$\mathrm{N}_{2}$} \\
\hline & . & - & . & - & $\mathrm{A}_{1}$ & \\
\hline & \multirow[b]{3}{*}{ الأسكوربيك } & . & . & . & $\mathrm{N}_{0}$ & \multirow{3}{*}{$\mathrm{S} \times \mathrm{N}$} \\
\hline & & - & - & - & $\mathrm{N}_{1}$ & \\
\hline & & . & . & - & $\mathrm{N}_{2}$ & \\
\hline & . & . & . & . & $\mathrm{A}_{0}$ & \multirow[b]{2}{*}{$\mathrm{S} \times \mathrm{A}$} \\
\hline & . & . & & & $\mathrm{A}_{1}$ & \\
\hline
\end{tabular}




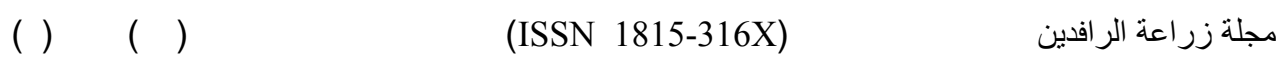

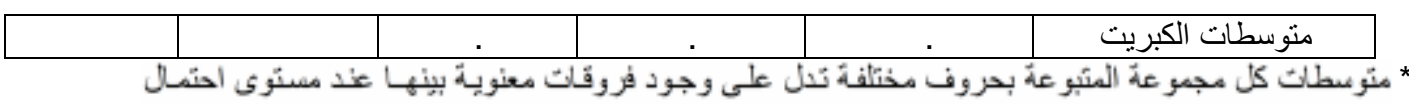

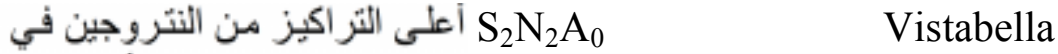

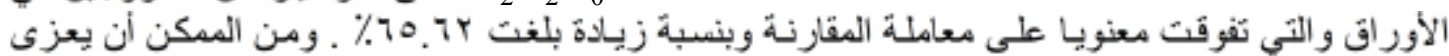

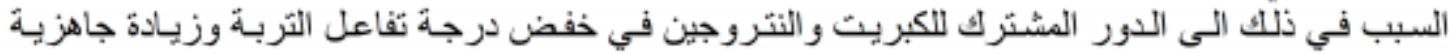

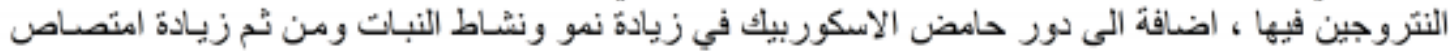

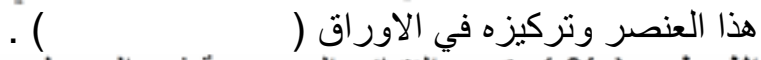

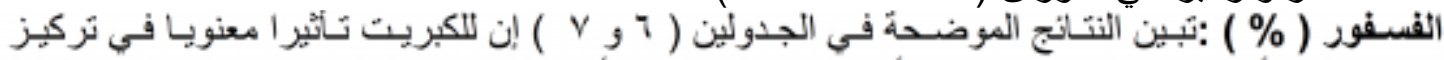

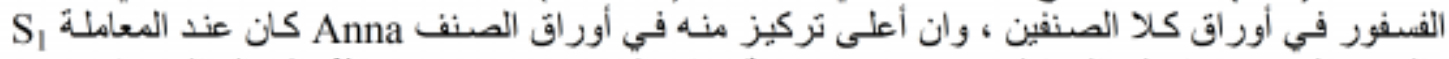

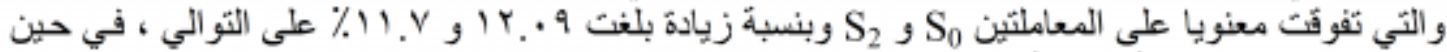

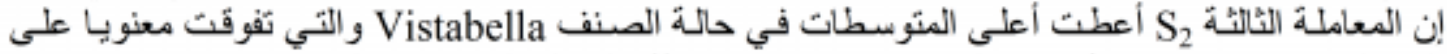

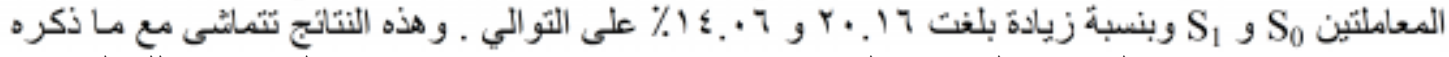

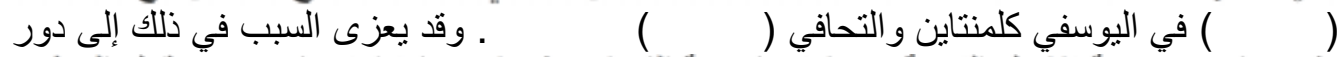

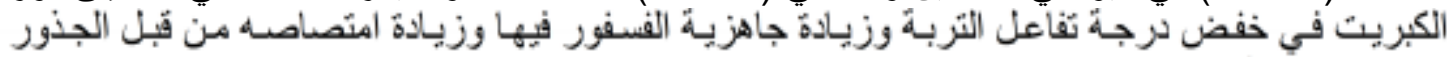

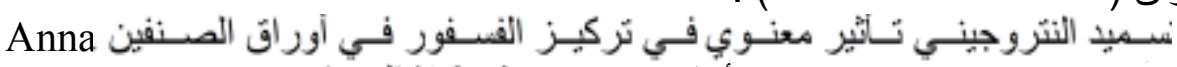

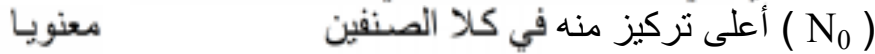

Vistabella ئ معاملتي

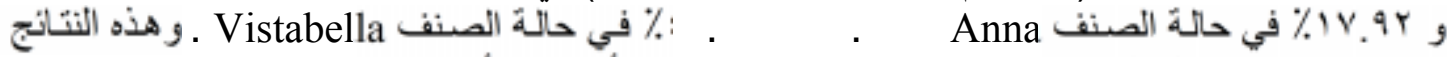

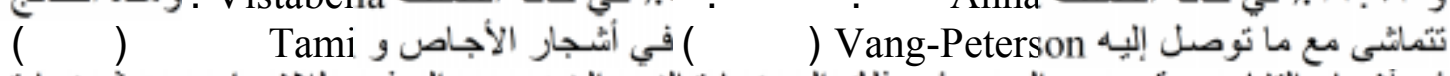

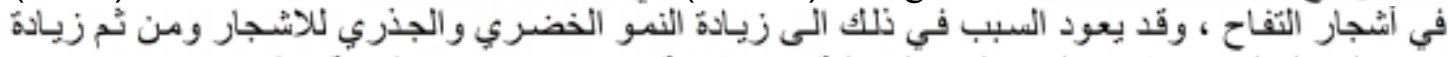

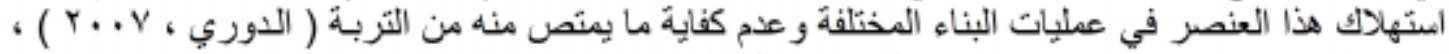

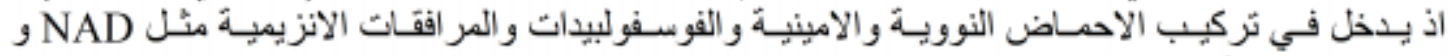

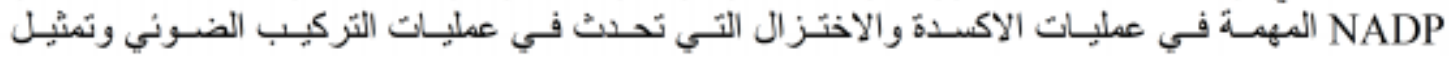

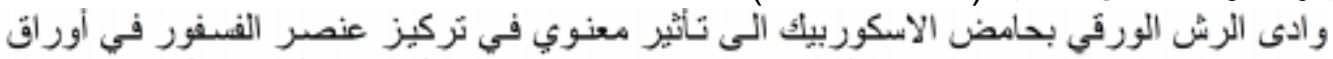
Anna

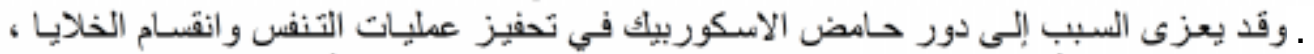

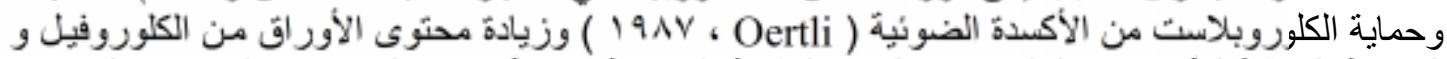

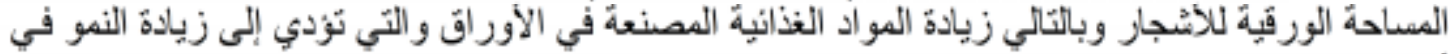

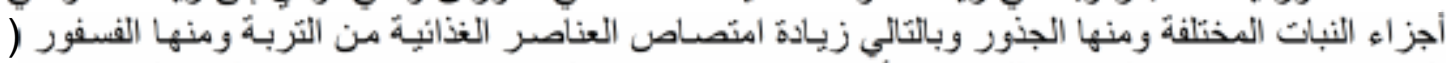

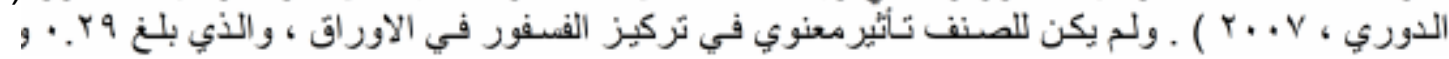

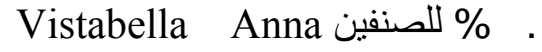

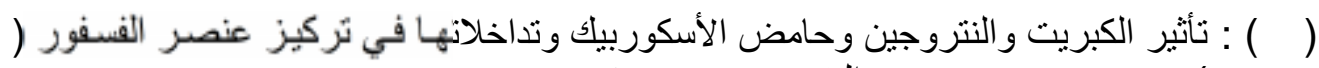

\begin{tabular}{|c|c|c|c|c|c|c|}
\hline \multirow{3}{*}{ النتروجين } & & & ana & & ( & \\
\hline & \multirow{2}{*}{$\mathrm{N} \times \mathrm{A}$} & \multicolumn{3}{|c|}{ الكبريت (S) } & \multirow{2}{*}{ الأسكوربي } & \multirow{2}{*}{$\begin{array}{c}\text { النتروجين } \\
\text { ( N ) }\end{array}$} \\
\hline & & $\mathrm{S}_{2}$ & $\mathrm{~S}$ & $\mathrm{~S}_{0}$ & & \\
\hline \multirow[t]{2}{*}{. } & . & . & . & . & $\mathrm{A}_{0}$ & \multirow[t]{2}{*}{$\mathrm{N}_{0}$} \\
\hline & . & . & . & - & $\mathrm{A}_{1}$ & \\
\hline & . & . & . & . & $\mathrm{A}_{0}$ & \multirow[t]{2}{*}{$\mathrm{N}_{1}$} \\
\hline & & & - & . & $\mathrm{A}_{1}$ & \\
\hline & . & . & . & - & $\mathrm{A}_{0}$ & \multirow[t]{2}{*}{$\mathrm{N}_{2}$} \\
\hline & . & . & . & - & $\mathrm{A}_{1}$ & \\
\hline & & . & 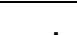 & & $\mathrm{N}_{0}$ & \\
\hline
\end{tabular}




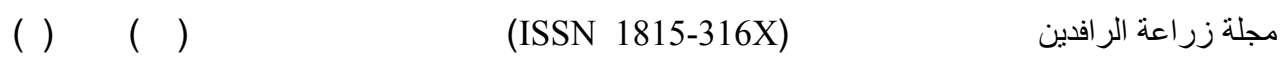

\begin{tabular}{|c|c|c|c|c|c|c|}
\hline & \multirow{2}{*}{} &. &. &. & $\mathrm{N}_{1}$ & $\mathrm{~S} \times \mathrm{N}$ \\
\cline { 2 - 6 } &. &. &. & $\mathrm{N}_{2}$ & \\
\hline &. &. &. &. & $\mathrm{A}_{0}$ & \multirow{2}{*}{$\mathrm{S}$} \\
\hline &. &. &. & $\mathrm{A}_{1}$ & $\mathrm{~S}$ \\
\hline & &. &. &. & \multicolumn{2}{|c|}{ * } \\
\hline
\end{tabular}

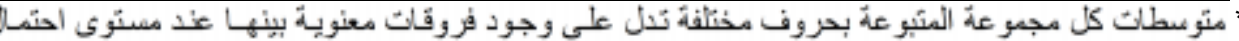

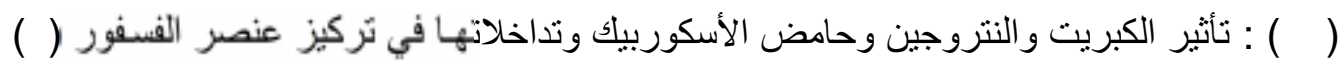

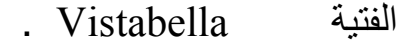

\begin{tabular}{|c|c|c|c|c|c|c|}
\hline \multirow{2}{*}{ النتروجين } & \multirow{2}{*}{$\mathrm{N} \times \mathrm{A}$} & \multicolumn{3}{|c|}{ الكبريت ( S ) } & \multirow{2}{*}{ الأسكوربي } & \multirow{2}{*}{ النتروجين } \\
\hline & & $\mathrm{S}_{2}$ & $\mathrm{~S}_{1}$ & $\mathrm{~S}_{0}$ & & \\
\hline \multirow[t]{2}{*}{. } & . & . & - & . د هـ & $\mathrm{A}_{0}$ & \multirow[t]{2}{*}{$\mathrm{N}_{0}$} \\
\hline & . & . & . & - & $\mathrm{A}_{1}$ & \\
\hline \multirow[t]{2}{*}{. } & . & لده & ده هـ & ده & $\mathrm{A}_{0}$ & \multirow[t]{2}{*}{$\mathrm{N}_{1}$} \\
\hline & . & هـ & ده & هـ & $\mathrm{A}_{1}$ & \\
\hline \multirow[t]{5}{*}{. } & . & . & هـ & . A ג & $\mathrm{A}_{0}$ & \multirow{5}{*}{$\mathrm{S} \times \mathrm{N}$} \\
\hline & . & . & ده & $\rightarrow$. & $\mathrm{A}_{1}$ & \\
\hline & \multirow[b]{3}{*}{ الأسكوربيك } &. & . & . & $\mathrm{N}_{0}$ & \\
\hline & & . & . & . & $\mathrm{N}_{1}$ & \\
\hline & & . & . & 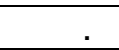 & $\mathrm{N}_{2}$ & \\
\hline & . & . & . & . & $\mathrm{A}_{0}$ & \multirow[b]{2}{*}{$\mathrm{S} \times \mathrm{A}$} \\
\hline & . & . & . & . & $\mathrm{A}_{1}$ & \\
\hline & & . & & & \multicolumn{2}{|c|}{ منوسطات الكبريت } \\
\hline
\end{tabular}

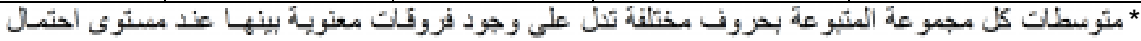

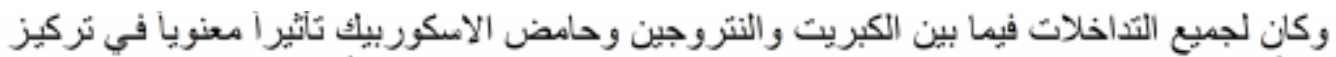

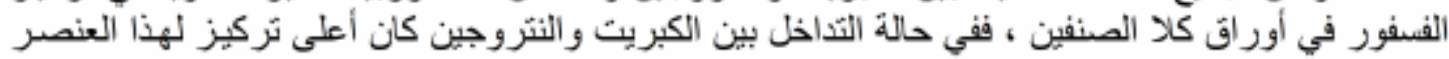
عند المعاملة

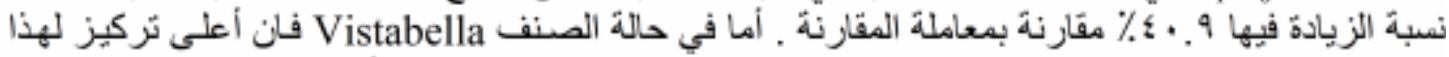

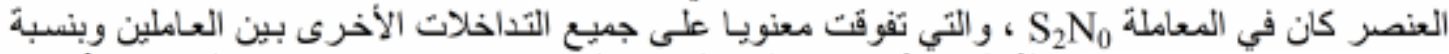

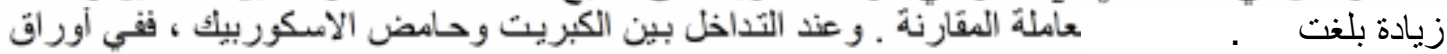

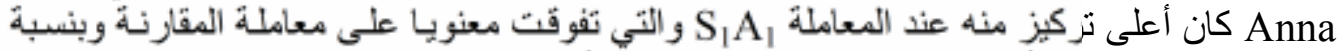

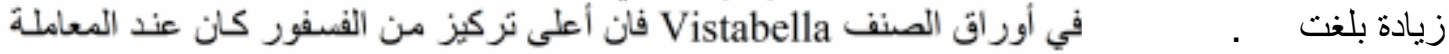

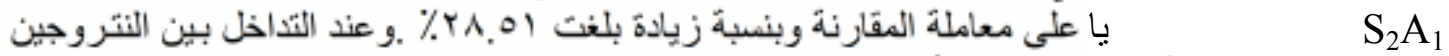

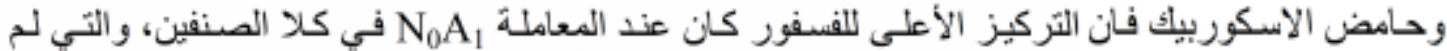

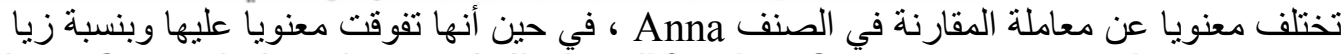

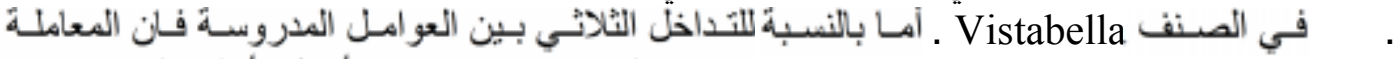
هistabella في الصنف $\mathrm{S}_{2} \mathrm{~N}_{0} \mathrm{~A}_{1} \quad$ Anna $\quad \mathrm{S}_{1} \mathrm{~N}_{0} \mathrm{~A}_{0}$

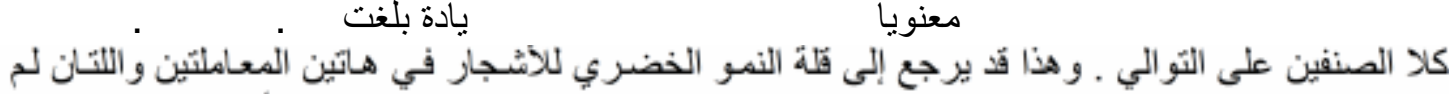

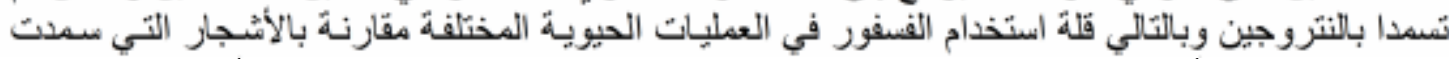

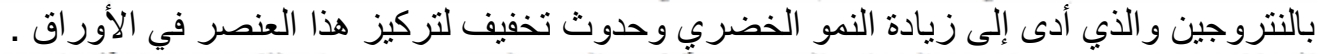

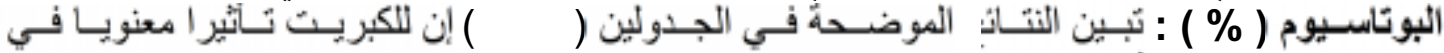

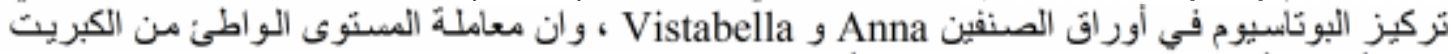

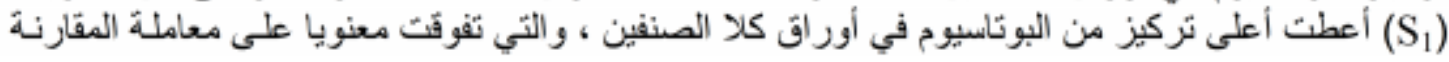



( ) ( )
(ISSN 1815-316X)

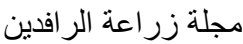

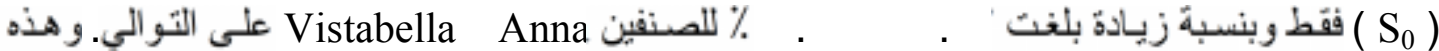

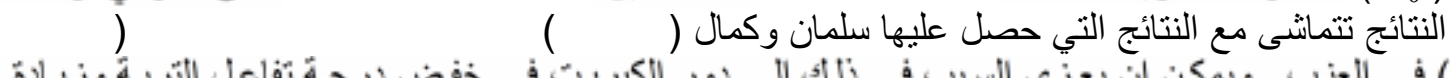

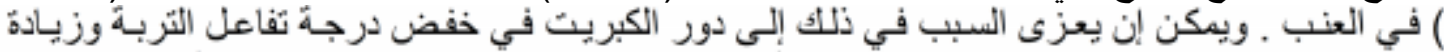

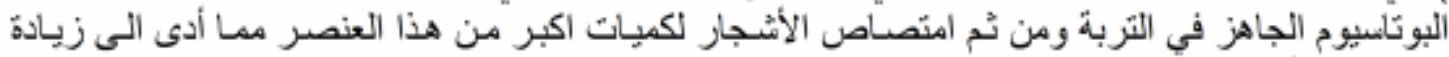
تركيزه في أور اقها ( Christensen

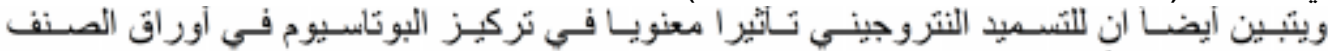
فistabella

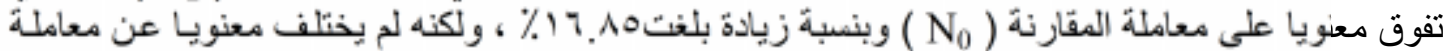

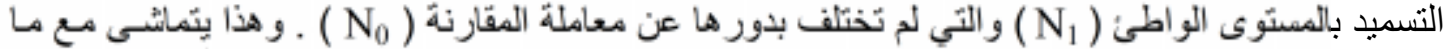

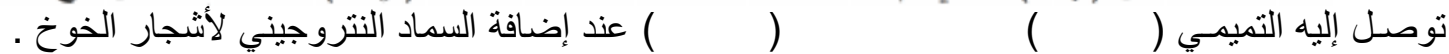

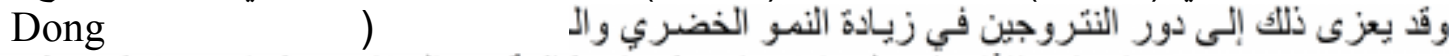

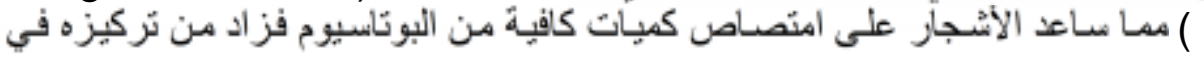

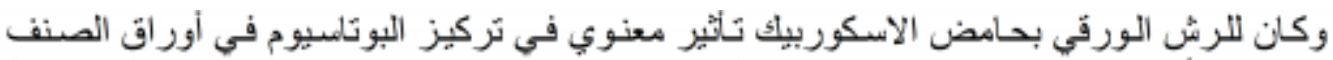
Anna

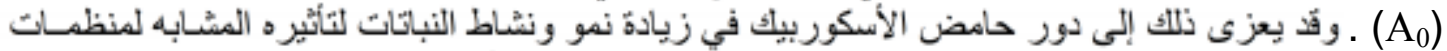

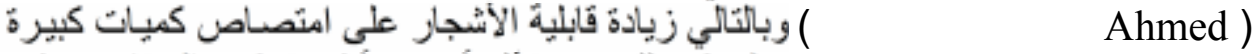

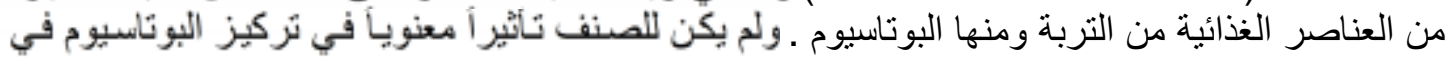

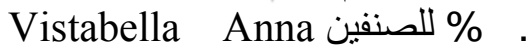

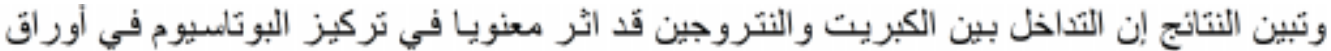
Vistabella

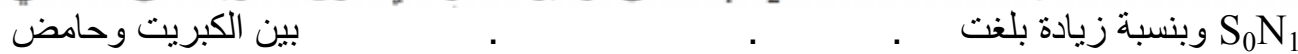
الاسكور بيك معنويا في تركيز البو تاسيو في أور اق كلا الصنفين ، وقد أعطت المعامل S S أعلى التر اكيز

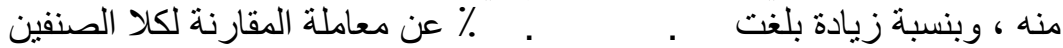

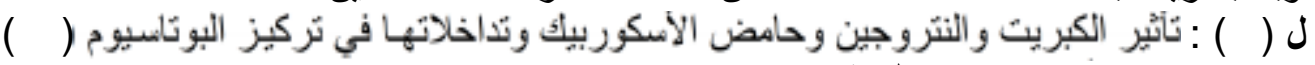

\begin{tabular}{|c|c|c|c|c|c|c|}
\hline \multirow{3}{*}{ النتروجين } & \multirow{3}{*}{$\mathrm{N} \times \mathrm{A}$} & \multicolumn{3}{|c|}{ Anna } & \multirow{3}{*}{ الأسكوربي } & \multirow{3}{*}{$\begin{array}{c}\text { النتروجين ( ) } \\
\text { ( ) }\end{array}$} \\
\hline & & \multicolumn{3}{|c|}{ الكبريت (S ) } & & \\
\hline & & $\mathrm{S}_{2}$ & $\mathrm{~S}_{1}$ & $\mathrm{~S}_{0}$ & & \\
\hline . & & & $\dot{x}$ & . & $\mathrm{A}_{0}$ & $\mathrm{~N}_{0}$ \\
\hline & & & . & . & $\mathrm{A}_{1}$ & \\
\hline . & & & . & . & $\mathrm{A}_{0}$ & $\mathrm{~N}_{1}$ \\
\hline & & . & . & . & $\mathrm{A}_{1}$ & \\
\hline . & & . & . & . & $\mathrm{A}_{0}$ & $\mathrm{~N}_{2}$ \\
\hline & & . & . & . & $\mathrm{A}_{1}$ & \\
\hline & & & . & . & $\mathrm{N}_{0}$ & \\
\hline & & & . & . & $\mathrm{N}_{1}$ & $\mathrm{~S} \times \mathrm{N}$ \\
\hline & الأسكوربيك & . & . & . & $\mathrm{N}_{2}$ & \\
\hline & . & . & . & . & $\mathrm{A}_{0}$ & \\
\hline & & & . & . & $\mathrm{A}_{1}$ & $\mathrm{~S} \times \mathrm{A}$ \\
\hline & & & . & . & لكبريت & متوسطا \\
\hline
\end{tabular}

*متوسطات كل مجمو عة المتبو عة بحروف مختلفة تدل على وجود فروقات معنوية بينها عند مستوى احتمال

( ) تأثير الكبريت والنتروجين وحامض الأسكوربيك وتداخلاتها في تركيز البوتاسيوم ( )

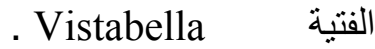

\begin{tabular}{|l|l|l|l|l|}
\hline & ( S ) النتروجين & & \\
\hline
\end{tabular}




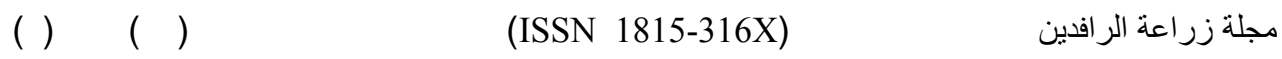

\begin{tabular}{|c|c|c|c|c|c|c|}
\hline النتروجين & $\mathrm{N} \times \mathrm{A}$ & $\mathrm{S}_{2}$ & $\overline{S_{1}}$ & $\mathrm{~S}_{0}$ & الأسكوربي & $(\mathrm{N})$ \\
\hline \multirow[t]{2}{*}{. } & . & - & - & . & $\mathrm{A}_{0}$ & \multirow[t]{2}{*}{$\mathrm{N}_{0}$} \\
\hline & . & - & - & - & $\mathrm{A}_{1}$ & \\
\hline \multirow[t]{2}{*}{. } & . & - & - & . & $\mathrm{A}_{0}$ & \multirow[t]{2}{*}{$\mathrm{N}_{1}$} \\
\hline & . & - &. & - & $\mathrm{A}_{1}$ & \\
\hline & . & - & . & - & $\mathrm{A}_{0}$ & \multirow[t]{2}{*}{$\mathrm{N}_{2}$} \\
\hline & & - & . & - & $\mathrm{A}_{1}$ & \\
\hline & \multirow{3}{*}{ متؤســـــــات } & . & - & . & $\mathrm{N}_{0}$ & \multirow{3}{*}{$\mathrm{S} \times \mathrm{N}$} \\
\hline & & . &. & . & $\mathrm{N}_{1}$ & \\
\hline & & . & . & . & $\mathrm{N}_{2}$ & \\
\hline & . & . & & . & $\mathrm{A}_{0}$ & \multirow{2}{*}{$\mathrm{S} \times \mathrm{A}$} \\
\hline & . & . & . & . & $\mathrm{A}_{1}$ & \\
\hline & & & . & 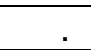 & \multicolumn{2}{|c|}{ متوسطات الكبريت } \\
\hline
\end{tabular}

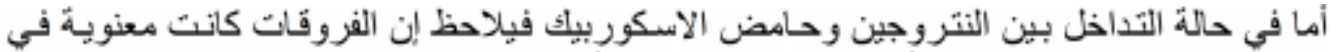

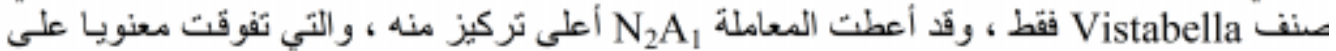

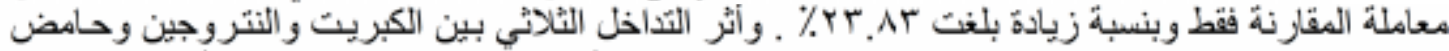

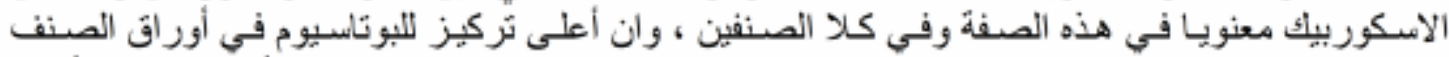
Anna

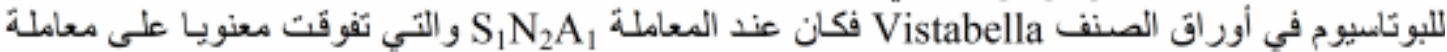

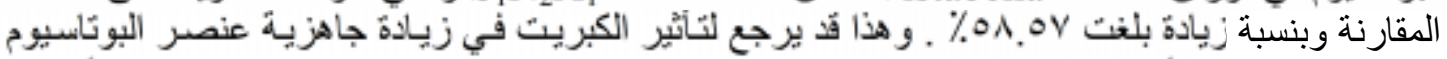

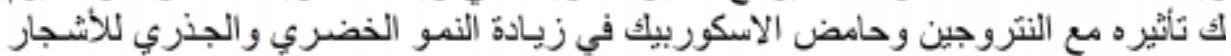

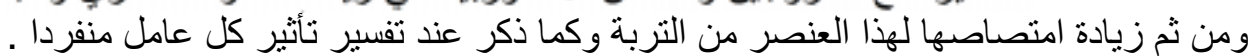

\section{EFFECT OF SULPHUR, NITROGEN AND ASCORBIC ACID ON MINERAL CONTENT OF YOUNG APPLE TREES CVS. ANNA AND VISTABELLA}

Jassim M. A. Al-Aa' reji

Hort.\&LandscapeDesignDept. CollegeofAgric\&Forstry, MosulUniv.,Iraq
Ehsan F. S. Al-Douri

Hort.Dept.Collge.Agric.

Tikrit Univ.Iraq.

\section{ABSTRACT}

Tow isolated experiments were conducted in the field of the College of Agriculture \& Forestry / University of Mosul, during the 2006 growing season . The first was on Anna cv. young apple trees and the second was on Vistabella cv. young apple trees which were grafted on $\mathrm{MM}_{106}$ rootstock, and planted in sandy clay lomy soil at $4 \times 4$ meters apart under drip irrigation system, to investigate the effect of sulphur, nitrogen, ascorbic acid and their interactions on leaves S, N, P and $\mathrm{K}$ concentrations. Three levels of each sulphur $\left(0,100\right.$ and $200 \mathrm{gm}$. tree ${ }^{-1}, \mathrm{~S}_{0}$, $S_{1}$ and $S_{2}$ respectively ), and nitrogen $\left(0,30\right.$ and $60 \mathrm{gm}$. tree ${ }^{-1}, \mathrm{~N}_{0}, \mathrm{~N}_{1}$ and $\mathrm{N}_{2}$ respectively) and two levels of ascorbic acid ( 0 and 125 mg.L. ${ }^{-1}, A_{0}$ and $A_{1}$ respectively) were used. The results of both experiments indicated that Sulphur application significantly increase leaves $\mathrm{S}, \mathrm{N}, \mathrm{P}$ and $\mathrm{K}$ concentration at both CVS. While nitrogen application leds to significant increase in leaves $\mathrm{S}$ and $\mathrm{N}$ 
concentration of both cultivars and $\mathrm{K}$ in leaves of Vistabella CV.and significant decrease of leaves $\mathrm{P}$ in both cultivars. Whereas ascorbic acid spray led to significant increase in leaves $\mathrm{N}$ in both cultivars and leaves $\mathrm{S}, \mathrm{P}$ and $\mathrm{K}$ in Anna cultivar.All interactions among sulphur, Nitrogen and ascorbic acid significantly effected leaves S , N , P and K concentrations on both cultivars, except the effect of S X N and N X Ascorbic acid interactions on K concentration in leaves of Anna CV. , and the effect of $\mathrm{S} X$ Ascorbic acid interaction on $\mathrm{S}$ concentration in leaves of Vistabella CV.The treatment $200 \mathrm{gm} \mathrm{S} . \mathrm{tree}^{-1}+60 \mathrm{gm} . \mathrm{N}$.tree ${ }^{-1}+$ foliar spray of ascorbic acid at $125 \mathrm{mg} . \mathrm{L}^{-1}$ was the best , the percentage of S , N , P and K on it was $0.34,2.94,0.295$ and $1.99 \%$ respectively in leaves of Anna CV.and $0.49,1.81$, 0.223 and $2.11 \%$ respectively in the leaves of Vistabella CV.

\section{المصادر}

المجموعة الإحصائية السنوية للفو اكه والخضر الجهزز المركزي للإحصاء ، وزارة التخطبط ،

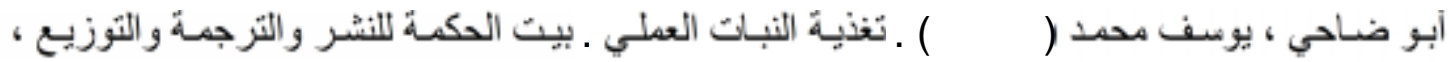

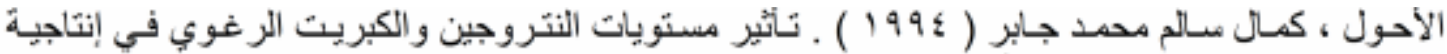

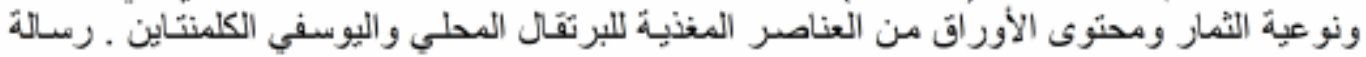

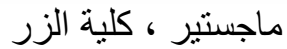

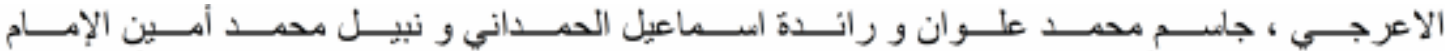

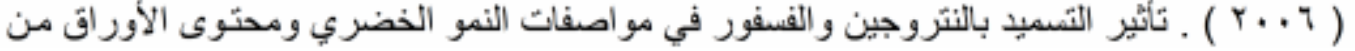

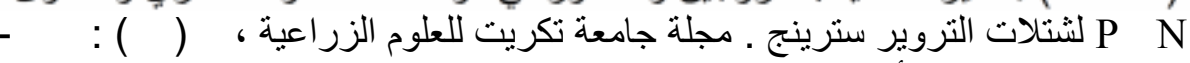

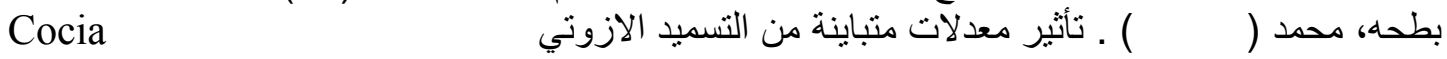

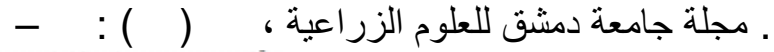

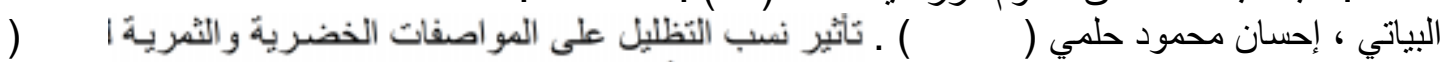

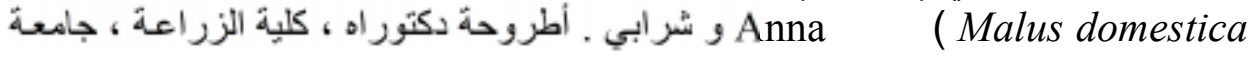

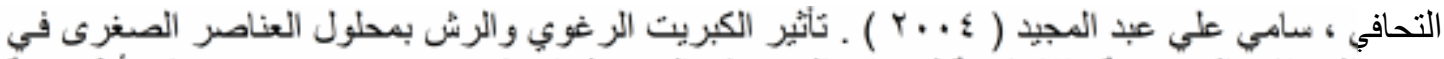

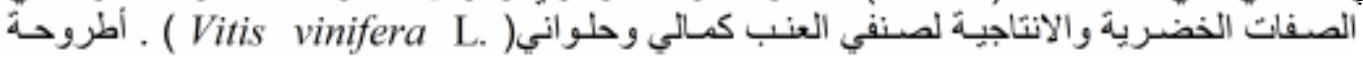

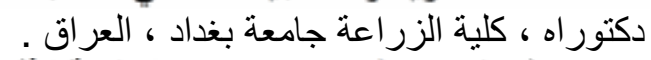

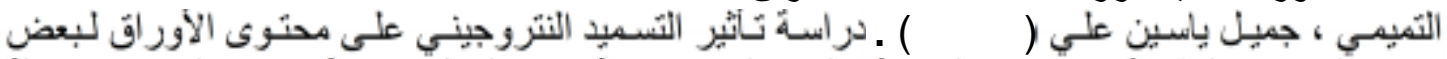

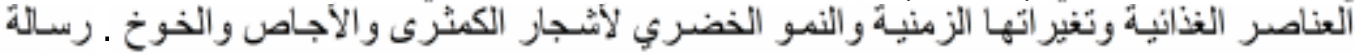

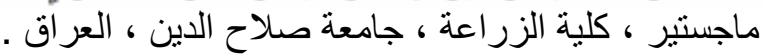

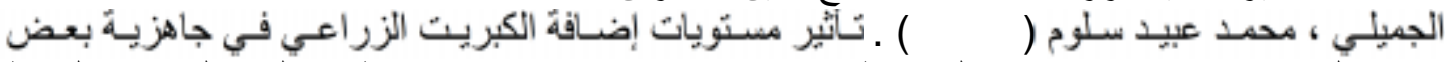

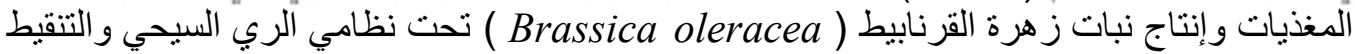

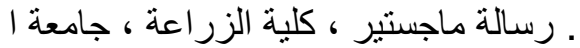

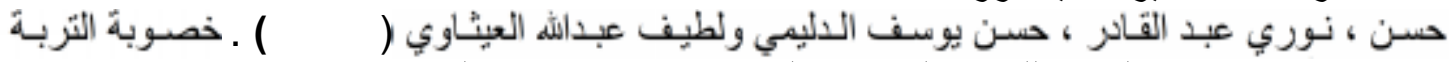

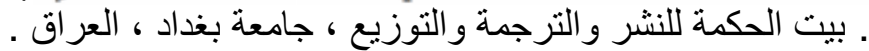

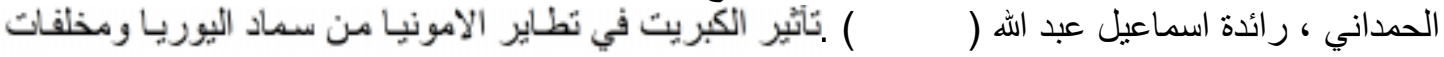

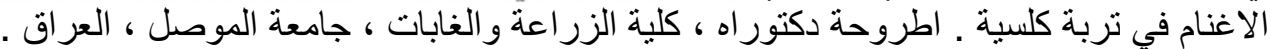

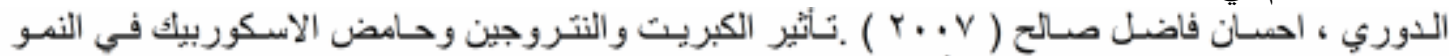

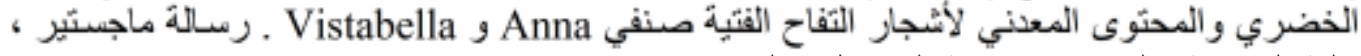

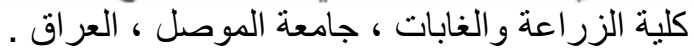




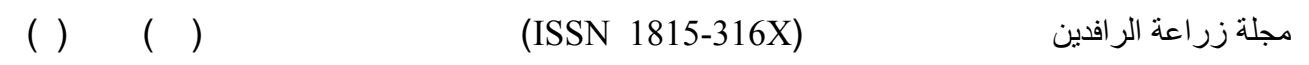

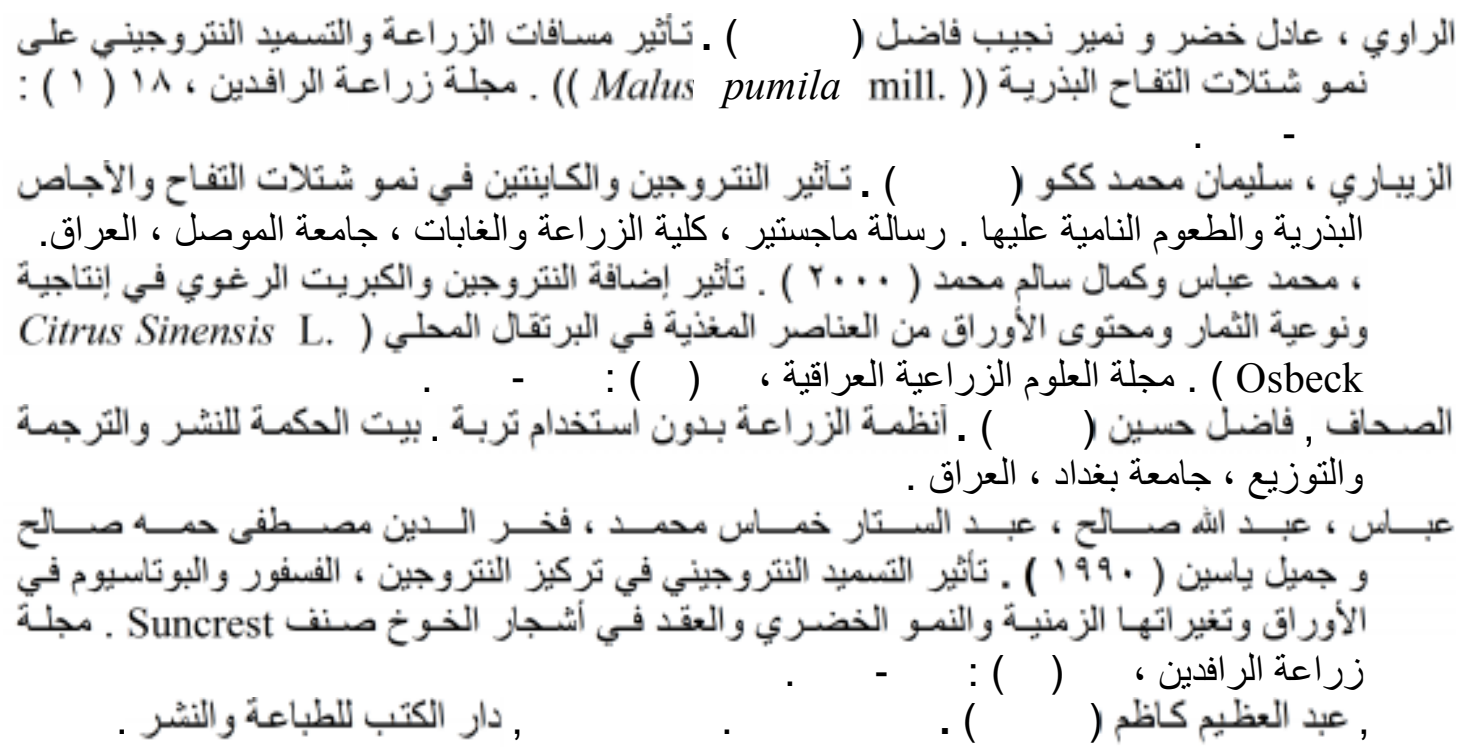

Ahmed, F. F.; M. A. Ragab; A. A. Ahmed and A. E. M. Mansour (1997A). Efficiency of spraying boron, zinc, potassium and sulphur as affected with application of urea for Anna apple trees ( Malus domestica L. ) . Egypt. J. Hort., 24 (1) : 75-90 .

Ahmed, F.F. ; A.M. Akl ; A.A. Gobora and A.E. Mansour ( 1997B ). Yield and quality of Anna apple trees ( Malus domestica L. ) in response to foliar application of ascorbine and citrine fertilizer . Eygpt J. Hort., 25(2) : 120-139 .

Al-Kanani, T; A. F. Makenzie; J.W. Fyles ; S. Chazala and I. P. Ohallora ( 1994 ). Ammonia volatilization from urea amended with lingo-sulfonate and phosphoroamide. Soil Sci. Amer. J., 58 : 224-248 .

Asselbergs , E. A. M. ( 1957 ). Studies on the formation of ascorbic acid in detached apple leaves . Plant Physiol., 32 ( 4 ):326-329.

Bal, J. S. ( 2005 ). Fruit Growing . 3th edt. Kalyani Publishers, New Delhi- 110002.

Bhargava, B.S. and H.B. Raghupathi ( 1999 ). Analysis of plant materials for macro and micronutrients . p: 49-82 . In Tandon, H.L.S. ( eds ). Methods of analysis of soils, plants, water and fertilizers . Binng Printers L- 14 , Lajpat Nagor New Delhi, 110024 .

Chaudhary, I.A. and A.H. Cornfield ( 1966 ). Analyst, Lond. 91, 528.

Cheng, L.; S. Dong and L. H. Fuchigami ( 2002 ). Urea uptake and nitrogen mobilization by apple leaves in relation to tree nitrogen status in Autumn . J. Hort. Sci. \& Biotechnology , 77 (1) :13-18.

Christensen, L. P.; W. L. Peacock and M. L. Bianchi ( 1991). Potassium fertilization of Thomson seedless grapevines using fertilizer sources under drip irrigation . Amer. J.Enol.Vitic., 42: 3 : 227-232 .

Dawood, F.A. ; H.S. Rahi; K.B. Hummudi and M.H.M. Jammel ( 1992 ) . Sulphur and organic matter relationship and their effect on the availability of some micronutrient and wheat yield in calcareous soil . Proc. Middle East Sulphur Symposium, 12-16 February, Cairo-Egypt . 

( ) ( )
(ISSN 1815-316X)
مجلة زر اعة الر افدين

Dong, S.; L. Cheng ; C. F. Scagel and L. H. Fuchigami ( 2003 ). Root damage affects nitrogen uptake and growth of young Fuji/M.26 apple trees . J. Hort. Sci. \& Biotechology ,78 (3) : 410-415.

Dong, S.; L. Cheng ; C. F. Scagel and L. H. Fuchigami ( 2005 ). Methd of nitrogen application in Summer affects plant growth and nitrogen uptake in Autumn in young Fuji/M.26 apple trees . Comm. Soil Sci. \& Plant Anal., 36 ( 11 \&12 ): 1465-1477.

Erez,M. ( 2000 ) . Temprate Fruit Crops in Warm Climates . Kluwer Acad. Publishers . Dordecht Bostan / London .

FAO STAT (2007). FAO Statistics Division, 8 March . Faostat.org .

Fenn, L.B.; and S. Miyamoto ( 1981) . Ammonia loss and associate reaction of urea in calcareous soil . Soil Soc. Amer. J., 45: 537-540.

Havlin, J. L. ;J. D. Beaton ; S. L. Tisdale and W. L. Nelson ( 2005 ) . Soil Fertility and Fertilizers $.7^{\text {th }}$ edt. Upper Saddle River ,New Jersey.

Intodia, S.K. and M.P. Sahu (1999) . Effect of sulfur fertilization on distribution of sulfur in alkaline calcareous soil of South Rajasthan . J. Indian Soc. Soil Sci. , 47 ( 3 ) : 442-445.

Johnson, C.M. and A. Ullrich (1959) . Analytical methods for use in plant analysis . Bull. Calif. Agric.Exo. No.766.

Johnson, J.R.; D. Fahy ; N. Gish and P.K. Andrews ( 1999 ) . Influence of ascorbic acid sprays on apple sunburn . Good Fruit Grower, 50 (13) : 81 - 83 .

Khattari , S . K . and F. Shatat (1996). Effect of ammonium sulfate rate and doses of application on yield and fruit quality of apple cv. Starkrimson . Dirasat Agric. Sci., 23 ( 2 ) : $84-88$.

Mansour, A.E.M. ( 1998 ). A comparative study between fertilizing Balady grapevines with sulfur-coated urea and urea the traditionally fast release nitrogen fertilizer . Egypt . J. Hort., 25 (1) : 43 - 53 .

Oertli, J. J. ( 1987 ) . Exogenous application of vitamins as regulators for growth and development of plant. Preview . Z. Planzenr Nahr. Bodenk $150: 375-391$.

Raese, J. T. and D.C. Staiff ( 1983 ) . Effect of rate and source of nitrogen fertilizers on mineral composition of D'Anjou pears . J. Plant Nutr., 6 ( 9 ) : 769 - 779 .

Rasmussen, P. E. ; R. E. Ramig ; R. R. Alkmavas and C. M. Smith (1975). Nitrogen - sulphur relation in soft white winter wheat . II . Initial and residual effect of sulphur application on nutrient concentration, uptake, and ( $\mathrm{N} / \mathrm{S}$ ) ratios . Agron. J., 67:224-228.

SAS (1996) . Statistical Analysis System , SAS Institute Inc. Cary Nc. 27511 , USA.

Sholeh, R.; D.B. Lefory and G.J. Blair ( 1997 ) . Effect of nutrients and elemental sulfur particle size on elemental sulfur oxidation and the growth of Thiobacillus thiooxidans . Aust. J. Agric. Res., 48 : 497-501 .

Tami, M.; P. B. Lombard and T. L. Righetti ( 1986 ) . Effect of urea nitrogen on fruitfullness and fruit quality of Starkspur Golden Delicious apple trees. J. Plant Nutr., 9 ( 1 ): 75-85.

Vang-Peterson, O. ( 1973 ) . Leaf analysis 1 . Content of nutrients in leaf dry matter in apple, pear, plum, cherry, black currant and red currant in relation to 
nitrogen, potassium and magnesium. Tidsskrift for Plant Eavol., 77 ( 3 ) : 393 398 . 